\title{
Social Roles and Baseline Proxemic Preferences for a Domestic Service Robot
}

\author{
Kheng Lee Koay • Dag Sverre Syrdal • \\ Mohammadreza Ashgari-Oskoei • \\ Michael L. Walters • Kerstin Dautenhahn
}

Accepted: 1 March 2014 / Published online: 10 April 2014

(C) The Author(s) 2014. This article is published with open access at Springerlink.com

\begin{abstract}
The goal of our research is to develop socially acceptable behavior for domestic robots in a setting where a user and the robot are sharing the same physical space and interact with each other in close proximity. Specifically, our research focuses on approach distances and directions in the context of a robot handing over an object to a user. Our present study consisted of two parts. Firstly, we carried out a large-scale survey trying to identify the internal structure between users' different social role expectations and the relationships between these and users' technology usage. Results from this study led to the development of a measure for preferred robot social roles. In the second part, this measure was used in a live human-robot interaction (HRI) study in a home setting, designed to create a baseline understanding of
\end{abstract}

The work described in this paper was conducted within the EU Integrated Projects LIREC (LIving with Robots and intEractive Companions, funded by the European Commission under contract numbers FP7 215554, and partly funded by the ACCOMPANY project, a part of the European Union's Seventh Framework Programme (FP7/2007-2013) under grant agreement n287624.

K. L. Koay · D. S. Syrdal $(\varangle) \cdot$ M. Ashgari-Oskoei

M. L. Walters $\cdot$ K. Dautenhahn

Adaptive Systems Research Group, School of Computer Science, University of Hertfordshire, Hatfield, UK

e-mail: d.s.syrdal@herts.ac.uk

\section{K. L. Koay}

e-mail: k.1.koay@herts.ac.uk

M. L. Walters

e-mail: m.l.walters@ herts.ac.uk

K. Dautenhahn

e-mail: k.dautenhahn@herts.ac.uk

Present Address:

M. Ashgari-Oskoei

Allameh Tabataba'i University, Tehran, Iran

e-mail: oskoei@atu.ac.ir human-robot Proxemics approach directions and distances for a Care-O-bot ${ }^{\circledR} 3$ based on these roles. In order to support users interpretation of the robots intentions in those tasks, the HRI studies used a simple LED light display panel. Findings indicate that, participants were comfortable with the robot approaching to the closest implemented distance $(0.5 \mathrm{~m})$. For the task requiring relatively more coordination, participants preferred the robot to approach from the front to a larger extent than for tasks requiring less coordination. The ability to identify the signals from the LED display also impacted how participants evaluated the robots behaviour. Users who had previous experience of interacting with robots differentiated less between approaches, and also evaluated tasks requiring more coordination with the robot more favourably. The findings in the short-term sample were strongly influenced by expectations as to the social role expectation they had of the robot, suggesting that social expectations impact proxemic interactions even if the robot platform is clearly not humanoid.

Keywords Human-robot interaction - Proxemics - Fetch and carry · Social roles · Survey · Experimental study .

Robot home companion

\section{Introduction}

This paper examines the relationship between user expectations, based on social roles, and proxemics preferences in an interaction with the Care-O-bot ${ }^{\circledR} 3$ service robot. The Care-O-bot ${ }^{\circledR} 3$ is a robot prototype intended for use as a general assistive device in domestic and other humancentred environments [41]. The development of the CareO-bot ${ }^{\circledR} 3$ is part of a growing trend in which robots are becoming more widespread in domestic environments, a 
trend which may lead to them playing and important role in the everyday lives of different groups of users in the future $[13,45,46]$. Because of this, researchers in humanrobot interaction (HRI) have turned their attention to investigate the impact of such systems on users [11,63], especially when performing tasks intended to assist people in their daily activities $[3,10,25,28,32,50,57]$. At the University of Hertfordshire (UH), we aim to develop robotic companions that are capable of providing both cognitive functions (such as reminders) and physical assistance (such as fetching and carrying objects) to their users [8]. A key motivation of this work is to support the development of a robotic companion that can provide a valuable service for elderly people wishing to live independently in their own home environment. This work faces many challenges, both in terms of technical implementation and also in terms of how the user experiences the behavior of the robot. In order to ensure that the behavior of the robot while carrying out its assistive tasks does not have a negative impact on the everyday life of its user, the robot should be user friendly, comfortable to use as well as predictable and safe to interact with. These requirements apply both in terms of direct interaction with the user as well as when carrying out other tasks in the same living space as the user. While these are reasonable requirements for robots that are marketed for purchase by private individuals, such as the Autom system [29], when applied to assistive technologies assigned through care agencies, for instance for elderly users [49], the impact such a presence will have also becomes a salient ethical issue $[47,51]$ as the prospective users may have less choice in their adoption. As such, safety and acceptability are of utmost importance. Note, while this article does not study robot safety directly, our aim is to improve safety in HRI scenarios by developing socially acceptable robot behavior. Such approaches that focus on social robot behaviour may complement other hardware or control solutions to robot safety, see e.g. Pervez and Ryu [42], De Santis et al. [12], and Herrmann and Melhuish [20].

The remainder of this article is structured as follows. Section 1.1 motivates the study of human-robot proxemics (HRP) which is a core research theme in studies developing safe and socially acceptable behavior for a mobile robot sharing a physical space with its users. Section 1.2 describes the importance of user expectations and social roles that users assign to robots based on their mental models, or their preconceptions of what they expect the robot to be. Section 1.3 argues for the need of baseline HRP investigations for a given robot platform designed for HRI scenarios. In order to increase a user's understanding of the robots intentions in our HRP scenarios the robot used light signaling, which is motivated in Sect. 1.4. Our overall research aims are summarized in Sect. 1.5. Section 2 presents the methodology of our work which consists of two stages. Results from a survey that developed a measure for preferred robot social roles are presented in Sect. 2.1. The second phase, consisting of HRI studies involving short-term and longterm user samples, are described in Sect. 2.2 in conjunction with associated research questions and concrete research hypotheses concerning HRP and the use of light signalling in robot-to-human handing-over scenarios. Section 3 provides results of the HRI experiments. Section 4 analyses the results from the HRI experiment in light of the original research hypotheses. Section 5 discusses the overall results and provides conclusions regarding results of our studies on social roles, proxemics preferences and light signaling to create socially acceptable robot behavior in a handing-over task.

\subsection{Human-Robot Proxemics}

When considering the impact of a mobile robot within a human-centered environment, its ability to move independently of human agency makes it distinctly different from other technologies adopted for assistive purposes.

This ability, which allows robots to achieve a greater degree of usefulness than other technologies in various settings, also means that the appropriate negotiation of personal and shared space is a unique problem facing such systems. The initial concern is, of course, the physical safety of the robot' users as well as that of third parties sharing the space in which the robot is operating [37]. However, inability to do this in a manner that is also socially acceptable, may also be a major hurdle to their adoption and use [21,40]. For example, if a robot were to generate loud, intrusive acoustic warning sounds whenever it approached a person, the user would be unlikely to perceive this robot as a socially acceptable companion, in particular for long-term use. Because of this, there has been much interest in HRP as a means to ensure that physical interactions with robots are as safe and comfortable as possible $[41,53,56]$. A robot that is capable of adopting and regulating socially acceptable distances with its users autonomously will be able to maintain a certain level of safety without the necessity of using explicit warning signals. As suggested by Takayama and Pantofaru [56], Syrdal et al. [53] and Koay et al. [34] there are several factors that impact participant preferences in terms of robot proxemics behavior.

Walters et al. [60] focus on the specific distances, and how these specific distances change dependent on context, robot appearance, direction of approach, and ability to directly control the robot. They propose a HRP framework where the influencing factors are squarely defined with the robot, its tasks and its interaction modalities. Takayama and Pantofaru [56], however, extend this approach and define the influencing factors in HRP according to three dimensions. The Robot and Contextual Dimensions are similar to the factors described by Walters et al., while the Human Dimen- 
sion highlight the impact of individual differences, such as personality, specific experiences or gender. The suggestion here is that one may examine experimentally aspects of each dimension empirically. On the other hand, the findings of Syrdal et al. [53,55] and Torta et al. [58] suggest that Robot or Contextual dimensions in themselves are dependent on human factors. Individual differences, such as participants' gender and personality or handedness traits may impact how participants understand the robot, which in turn will impact the expectations that they have of it. However, these same differences in personality traits may also impact the evaluation of violations of these expectations as well.

In addition, personality also influences participants assumptions regarding aspects of the context, such as physical setting and task domain. For instance, participants with higher scores in extraversion may find social settings less stressful and problematic than participants with lower scores. Participants with a higher degree of conscientiousness may pay more attention to a task than participants with lower scores, which may in turn impact evaluations of the interaction [29].

Thus, all three dimensions suggested by Takayama and Pantofaru [56] will interact with each other in a manner that will vary from participant to participant, robotic platform to robotic platform, and situation to situation. It will be very difficult to disentangle them from each other, especially as they may also interact with the habituation effects reported by Koay et al. [34]. Because of this, it may be beneficial to use measures to directly assess expectations that the participants have of the robot and the interaction directly. One way that this can be achieved is to examine the expectations arising from the social role that the participants will assign to the robot within each anticipated interaction.

\subsection{Social Role Expectations}

For human-human interactions at least, this emphasis on social roles is in accord with the literature on proxemics. Both in terms of Hall's [18] interpersonal distances as well as Kendon's [26] spatial groupings, it is the situations and the relationships between people that are the most important in determining these behaviours. Personality traits or other idiosyncratic factors do play a role in terms of participants' actual preferences, in particular regarding experiences of personal space violations. However, it is a small role compared to situational modifiers, or relationships, relative status, gender and other external constraints [19]. This suggests that, for HRP, the perceived social role and status of the robot within the interaction needs to be considered. Indeed, peoples expectations of robots have been shown to impact interactions $[38,39]$. Therefore, due to the relationship between social role and HRP preferences as suggested by the recent work by Kim et al. [30], and Choi et al. [6], the prospec- tive users mental model of the robot in terms of social role expectations needs to be assessed.

Ljungblad et al. [39] highlight the temporal aspects of these mental models and how they change across sustained interactions, in particular, in terms of how they correspond to the actual robots in question. In addition, Koay et al. [34] demonstrated that changes occur in terms of proxemics HRP preferences as well. This may be due to participants' increasing understanding of the robots true capabilities and common behaviours with greater exposure and as their mental model of the robot more closely resembles the real robot (capabilities) over time.

\subsection{Establishing Baselines}

The issues raised in Sect. 1.1 (i.e. HRP) suggest the need to establish platform specific baselines for the specific contexts and tasks that arise from the capabilities of a particular robot. Baseline, in the context of our work, thus comprises the default parameters of robot behavior that are socially acceptable and preferred by users. It is important to identify such baseline parameters for any given specific robot platform, in our present study the Care-O-bot ${ }^{\circledR} 3$.

The Care-O-bot ${ }^{\circledR} 3$ which is described in more detail in Sect. 2.2, was designed and built by the Fraunhofer IPA (Stuttgart, Germany). The overall hardware and HRI design was inspired by the metaphor of an interactive butler robot $[41,44]$. It is a human-sized but non-humanoid robot that is radically different from most current robots in that it has two ends; a working end (i.e. with a lightweight industrial manipulator) and a presentation end (i.e. with a folding tray) that can be used to present objects to the user. This unique design configuration, and the safety concerns arising from the industrial arm working in a domestic, human-centred space [17], necessitates the creation of specific HRP baselines for this particular robot. This will allow for the specification of a user-friendly way for the robot to approach, position itself and interact with its users. The establishment of these baselines will thus address the basic HRI requirements necessary for it to be able to perform tasks in a userfriendly way by taking account of its users' preferences and expectations.

The design and capabilities of this platform suggest many possible interactions within the context of handing over objects. As in previous studies the focus will be on the role of approach direction and approach distance [1,36,61,62] and handing over style [33], based on the constraints of two particular types of handing-over scenario based on the specific affordances of the Care-O-bot ${ }^{\circledR} 3$ robot platform. This HRP baseline, along with how it is influenced by participants expectations based on the robots social role, will form a basis for ensuring that the Care-O-bot ${ }^{\circledR} 3$ performs its tasks in a socially acceptable manner. 


\subsection{Understanding the Robot's Intentions Via Explicit Signalling}

While user expectations and preferences, that arise from the context, platform and social role expectations, can be considered important implicit expectations, there may still be ambiguity which reduces the predictability of the robots behaviour. While humans, and to some extent humanoid robots, have quite a wide range of modalities through which they can signal their proxemic intentions or mitigate violations of proxemic expectations [4], the Care-O-bot ${ }^{\circledR} 3$ can be termed an appearance-constrained robot [2]. By this, we mean that its appearance is highly constrained by the physical tasks that it is expected to do, as opposed to robots that are intended purely for social HRI tasks, such as KASPAR [9], Geminoids [22], Paro [59], or toys like the AIBO or Pleo [14,23]. Robots that are constrained in terms of appearance may have to rely on explicit signalling, which sometimes may draw on animal behaviour [31,54], but are often presented as arbitrary signals, possibly drawing on signalling conventions (e.g. derived from traffic rules [2]) to communicate and disambiguate their intentions. The Care-O-bot ${ }^{\circledR} 3$ has a LED display panel which can be used to provide a simple and identifiable feedback signal to facilitate user interaction and safety. We propose that the use of simple, colour-coded LED displays can alert the user to ambiguous behaviour which might be potentially hazardous. In this study we used these LEDs to signal the main types of behaviour which the robot is currently engaged in. Of interest is both the ability of participants to notice and interpret these signals, and the impact on participant proxemic preferences. Also of interest is how participants conceptions of the robot in terms of social role expectations might influence this. Note that we are using light signalling displays for the robot to express its behavioural state in order to provide the user with information regarding what the robot is currently doing and when it is useful or safe to approach or to initiate interactions with the robot. We are not using light signalling for expressing emotional states or interaction history, which is a different direction of work (cf. Syrdal et al. [54] and Bethel and Murphy [2]).

\subsection{Research Aims}

The work presented in this paper has the following aims to advance previous work on proxemic preferences in handingover tasks:

1. Examine what high-level social roles expectations participants have of the robot.

2. Establish platform-specific HRP baselines for handingover tasks for the Care-O-bot ${ }^{\circledR} 3$.

3. Examine the viability of simple light-signals for disambiguating Care-O-bot ${ }^{\circledR} 3$ intentionality.
4. Examine the relationship between users' social role expectations and preferences regarding HRP as well as interpretation of LED signalling This will allow for an understanding of how participant social role expectations impact low-level proxemic expectations, which will increase the generalisability of results beyond the CareO-bot ${ }^{\circledR} 3$ platform.

In order to achieve these aims we initially surveyed the different roles that participants might assign to the robot, and examined whether or not there is an underlying structure to these roles. We subsequently conducted a study with the Care-O-bot ${ }^{\circledR} 3$ in a real setting to determine participant baseline proxemic preferences in a handing-over context. We also examined whether or not these preferences changed through long-term exposure to robots performing similar tasks, as suggested in Koay et al. [34]. This allowed us to meaningfully investigate how participants social role expectations impacted their proxemic preferences, not only in an initial HRI, but also in an interaction taking place after sustained interactions with robots. The use of explicit signalling to disambiguate robot intentions and the relationship between their efficacy, participant proxemics preferences and social roles were also examined.

\section{Methodology}

This study was conducted in two stages. The first concerned the development of a measure for preferred social roles for the robot. This involved a large-scale survey, which looked for internal structure between the participants different social role expectations and the relationships between these and their technology usage. This measure was then used in the second part, which was an HRI study designed to create a baseline understanding of HRP approach directions and distances for a Care-O-bot ${ }^{\circledR} 3$ [41] based on these roles. This HRI study was performed with two samples; The short-term sample who interacted with the Care-O-bot ${ }^{\circledR} 3$ only in this particular interaction, while the long-term sample had been involved in a long-term study with other mobile robots prior to the HRI study.

\subsection{Measurements-Survey}

The initial part of this investigation focused on examining the measurement of expected social roles for a robot and was a survey-based study. The participants were visitors at the Science Gallery in Dublin, Ireland who were asked to complete a brief survey which was run on an unattended computer. This study took part over a period of several months in the spring/summer of 2011 as part of the HUMAN+ exhibi- 
tion. ${ }^{1}$ The HUMAN+ exhibition was an exhibition that examined future possibilities in fields such as genetics as well as robotics. The exhibition featured several different robots. The computer used for this survey was placed near an exhibit titled My Robot Companion, created by the artists Anna Dumitriu and Alex May in collaboration with researchers from the UH [64]. The questionnaire itself consisted of two parts: The first part included demographic information including age and gender, as well as a question on participants computers use. The second part consisted of the statement: 'If you were to have a robot, would you like to interact with it as a...', followed by five different possible social roles. The participants were invited to rate their agreement/disagreement on 5 point Likert scale. These roles were chosen on the basis of the work by Ljungblad et al. [39] and Dautenhahn et al. [11], but retained a strong focus on roles pre-supposing long-term interactions. While there was a variety of robots and robotic installations within the interaction, no further guidance as to what sort of robot was being referred to was given to the participants. The roles suggested were as follows:

- Friend

- Servant

- Pet

- Colleague

- Tool

\subsubsection{Survey Results}

While some preliminary results regarding social role expectations have been published in [64], the following analysis is for the purpose of examining the underlying structure of these social roles, and their demographic correlates, in order to assess their validity as a measure for HRI studies.

\subsubsection{Survey Demographics}

The sample for the survey consisted of 211 males and 214 females. The mean age was 24.8 years. The majority of participants, however, clustered around the age of 20. In terms of computer usage, the mean hours spent using a computer a week was $21 \mathrm{~h}$, with a median of $15 \mathrm{~h}$. In terms of what the participant used computers the most for, Table 1 suggests that the most common usage of computers was for professional use, with social media and email use coming second, and the use for games coming third.

\subsubsection{Social Roles}

The initial correlation matrix between the Likert ratings of the different social roles can be found in Table 2 .

\footnotetext{
${ }^{1} \mathrm{http} / / /$ sciencegallery.com/humanplus/exhibits/.
}

Table 1 Computer usage in survey

\begin{tabular}{lcl}
\hline Category & No. of participants & Percentage \\
\hline Social media and email & 114 & 27.5 \\
Games & 63 & 15.2 \\
Work or school & 180 & 43.4 \\
Hobbies & 20 & 4.8 \\
Other & 38 & 8.2 \\
\hline
\end{tabular}

Table 2 Correlation matrix between social role ratings

\begin{tabular}{lccccc}
\hline & Friend & Servant & Pet & Colleague & Tool \\
\hline Friend & 1.00 & & & & \\
Servant & $-.74^{*}$ & 1.00 & & & \\
Pet & $.23^{*}$ & $.21^{*}$ & 1.00 & & \\
Colleague & $.62^{*}$ & -.07 & .19 & 1.00 & \\
Tool & $-.33^{*}$ & $.33^{*}$ & .04 & $-.23^{*}$ & 1.00 \\
$* p<.05$ & & & & &
\end{tabular}

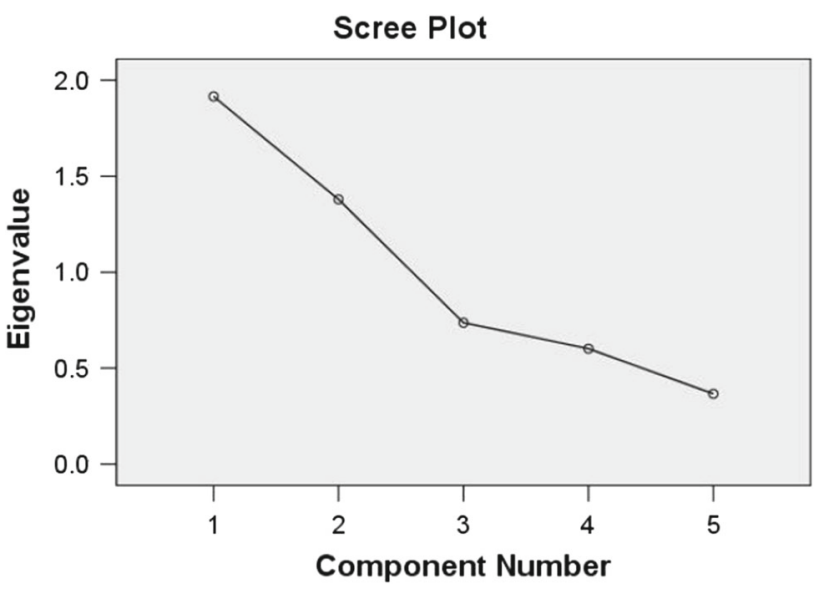

Fig. 1 Scree plot from PCA

Based on this correlation matrix, a Principal Components Analysis was performed. The initial run used the Kaiser extraction criteria [24] which suggests only retrieving components with an eigenvalue above 1 . This analysis returned 2 factors with an eigenvalue larger than one. Somewhat problematic however, was the rating of the "Pet"-item which loaded equally on both variables. This led to a re-examination of the components using the Cattell extraction criteria [5], which suggests that visually assessing the scree-plot (Fig. 1) and choosing the point at which the slope "evens out" allows for a better representation of the underlying structure of the data. This Scree Plot is described in Fig. 1 and suggests that three factors might be a better way of representing the structure of the data.

The varimax rotated 3 -factor solution can be found in Table 3 and suggests that there are three dimensions: The first dimension was tentatively called Equality as the variables Friend and Colleague loaded on this dimension. A high 
Table 3 Rotated factor loadings

\begin{tabular}{lccc}
\hline Variable & Equality & Comp control & Petlike \\
\hline Friend & $.86^{*}$ & -.17 & .17 \\
Servant & .02 & $.81^{*}$ & .24 \\
Pet & .13 & .09 & .97 \\
Colleague & $.91^{*}$ & -.04 & .03 \\
Tool & -.23 & $.81^{*}$ & -.12
\end{tabular}

*Loads on factor

score on this would suggest that the participant expected to have the robot act in a manner suggesting an equal (social) footing to themselves within interactions, while a low score would suggest the opposite (i.e. that the robot adopts a more deferential role).

The second factor was tentatively called Control as the variables Servant and Tool load on this factor. A high score on this dimension would suggest that the participant expects the robot's social role to be one in which the user will exert a high degree of control, while a lower score would suggest that the robot is expected to act in a more autonomous manner.

The third dimension deals almost solely with the Pet variable. This suggests that interactions associated with pets are not fully covered by our expectations in terms of equality and control. However, this third variable explains less than the variance of one of the items. It is also important to note that they are positive expectations. A high score along any of these dimensions suggest that a participant expects and would like to interact with a robot in this manner.

\subsubsection{Computer Usage}

The relationship between computer usage and the preferred social roles for a robot, was assessed by a series of ANOVAs. Only the three categories, Work/School, Social Media/Email and Games were included in the analyses. The group "Other" was excluded for reasons that it would be too heterogeneous in terms of computer usage, and the group "Hobbies" was excluded due to its small size. The results of this analysis are described in Table 4 and Fig. 2, which both point towards three main effects. The first main effect was found for Computer Usage on the equality dimension $\left[F(2,329)=10.46, p<.001, \eta^{2}=.06\right]$. This effect suggests that participants that use computers primarily for games were more likely to consider and prefer the robot to act as more of an equal within the interaction. Another effect was also found for Computer Usage on the control dimension $\left[F(2,330)=3.25, p<.05, \eta^{2}=.02\right]$ suggesting that participants who use computers the most for work were more likely to consider and prefer the robot to be something to be controlled, compared to the other participants. The final significant effect was found for Computer Usage on the Petlike dimension $\left[F(2,331)=3.15, p<.05, \eta^{2}=.02\right]$. This
Table 4 Computer usage and social roles

\begin{tabular}{llll}
\hline Computer usage & $\begin{array}{l}\text { Mean equality } \\
(\mathrm{SE})\end{array}$ & $\begin{array}{l}\text { Mean control } \\
(\mathrm{SE})\end{array}$ & $\begin{array}{l}\text { Mean petlike } \\
(\mathrm{SE})\end{array}$ \\
\hline Social media & $2.34(.11)$ & $3.55(.09)$ & $2.65(.13)$ \\
Games & $3.04(.15)$ & $3.46(.14)$ & $2.21(.18)$ \\
Work/school & $2.25(.09)$ & $3.80(.08)$ & $2.28(.10)$ \\
\hline
\end{tabular}

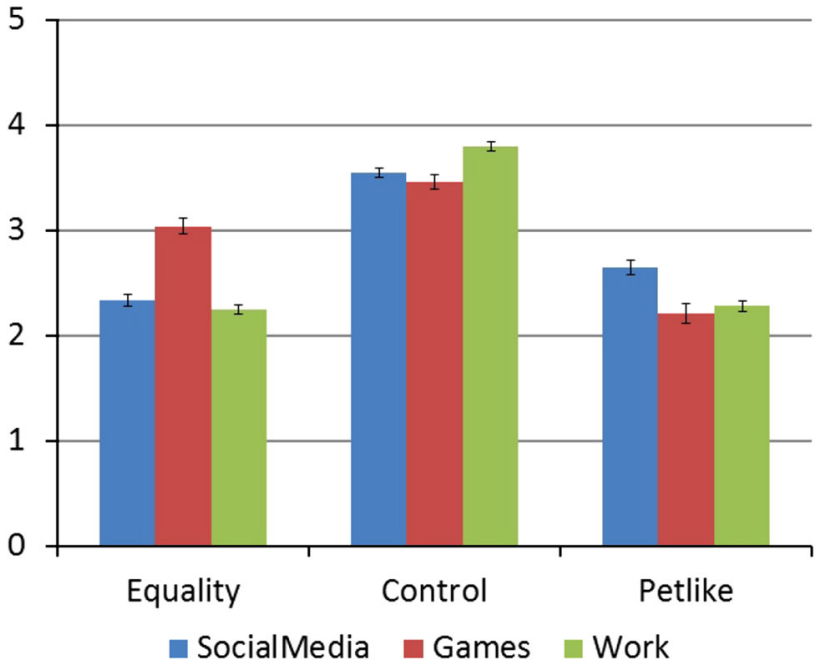

Fig. 2 Relationship between computer usage and social roles

effect suggests that participants that use computers for mostly Social Media and Email, preferred the role of the robot to be more pet-like compared to other participants.

\subsubsection{Comment on the Measures}

The survey was successful in establishing the structural relationship between the five items. It also suggested that there were systematic relationships between how participants viewed the preferred social roles of robots and how they interacted with technology. Participants using computers primarily to play games, see interactions between themselves and a possible robot as similar to those they would have with equals. Such interactions would be more social in nature and also more collaborative. It is possible that the exposure to collaboration and competition with characters controlled by artificial intelligence within computer games may have led to this result. It is also possible that engaging in intrinsically rewarding interactions with computational artefacts may also have led to an interest for interactions that are more social in nature. In terms of control, participants who use the computer the most for work or academic purposes are more likely to score highly along this dimension. One likely explanation for this is the need for exact control and efficiency of the computer's behaviour in these settings, unlike in games-usage where entertainment is not necessarily 
related to efficiency. Also, this use of computational artefacts is for extrinsic rewards and so might be associated to expectations for interactions that are more task oriented. The final dimension, pet-like, seems to be more popular amongst users that use computers primarily for social purposes. Pets traditionally have had a dual purpose, having an intrinsic social value as well as value in terms of services that can be performed by them [7], and this might be reflected in this group of computer users. Note, the effect size of these relationships, as suggested by the $\eta^{2}$ measure for all the above effects, were quite small, and only discernible in a large sample. Despite this, the above results suggest that the three dimensions of social expectations of interactions with robots had a systematic relationship with participants' previous interactions with other information technology artefacts which lent support to the notion of using them in a live HRI study, in order to examine their impact on interaction preferences.

\subsection{Human-Robot Interaction Study}

The task context used in this study was based on a fetch and carry scenario where the Care-O-bot ${ }^{\circledR} 3$ could be used to support participants in their daily activities by providing an object fetching functionality.

\subsubsection{Setting: University of Hertfordshire Robot House}

The study was conducted at the UH Robot House, which is dedicated to HRI Studies in an ecologically valid domestic environment as compared to laboratory conditions. The $\mathrm{UH}$ Robot House has two floors, four bedrooms and is a fully furnished British house. Only the living room was used for this study. Figures 3 and 4 show the trial setup, indicating the initial locations of the robot, participant, experimenter and relevant objects within the trial area.

\subsubsection{Robot}

The robot used in this study was the human-scaled but nonhumanoid Care-O-bot ${ }^{\circledR} 3$ [41] (Fig. 5). It has the capability of omni-directional navigation and is equipped with a highly flexible, commercial arm with seven degrees of freedom as well as with a three-finger hand to support fetch and carry tasks. It has a tray to serve objects and a touch screen panel to facilitate user interaction. The deliberately chosen nonhuman appearance was designed by a professional team of designers. In order to reduce anthropomorphic attributions, which have been shown to lead to unrealistic expectations of users in HRI, any specific parts that resemble a face or head, or produce gender specific expressions were avoided [15].

The Care-O-bot ${ }^{\circledR} 3$ supports basic body gestures like bowing or nodding and is capable of utilising LED light display signals, sound and speech to provide feedback to the user. It

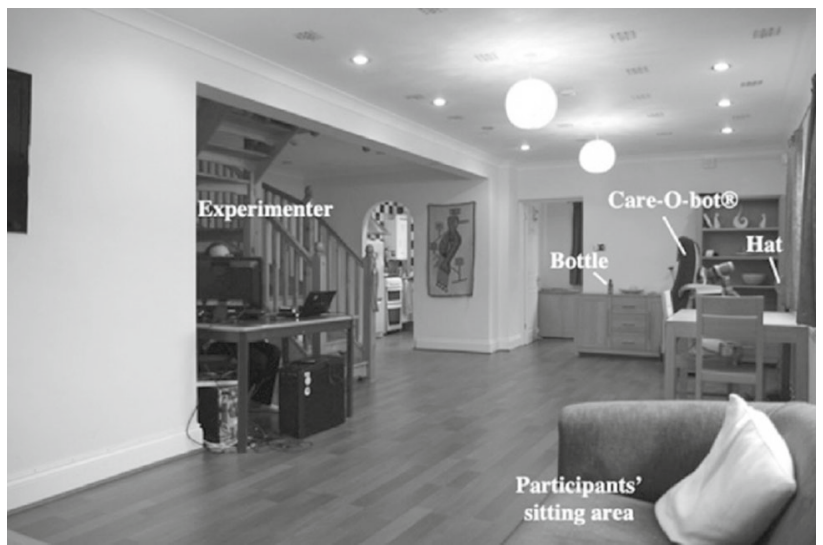

Fig. 3 The setup and locations of the robot and participant, experimenter and objects within the trial area (Photo)

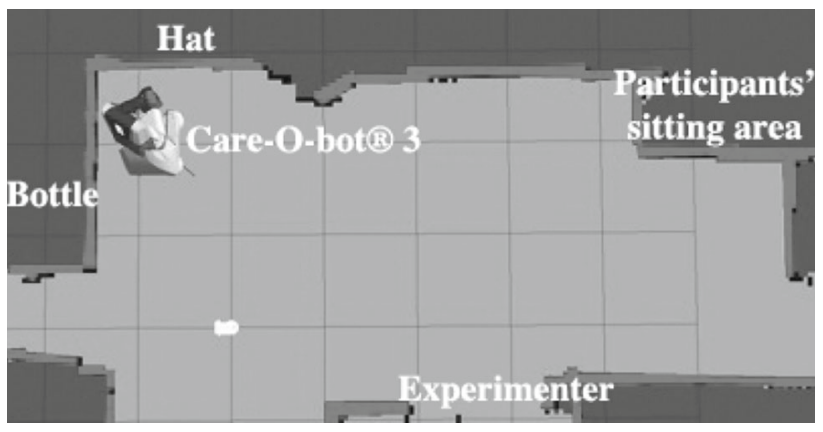

Fig. 4 The setup and locations of the robot and participant, experimenter and objects within the trial area (Map)

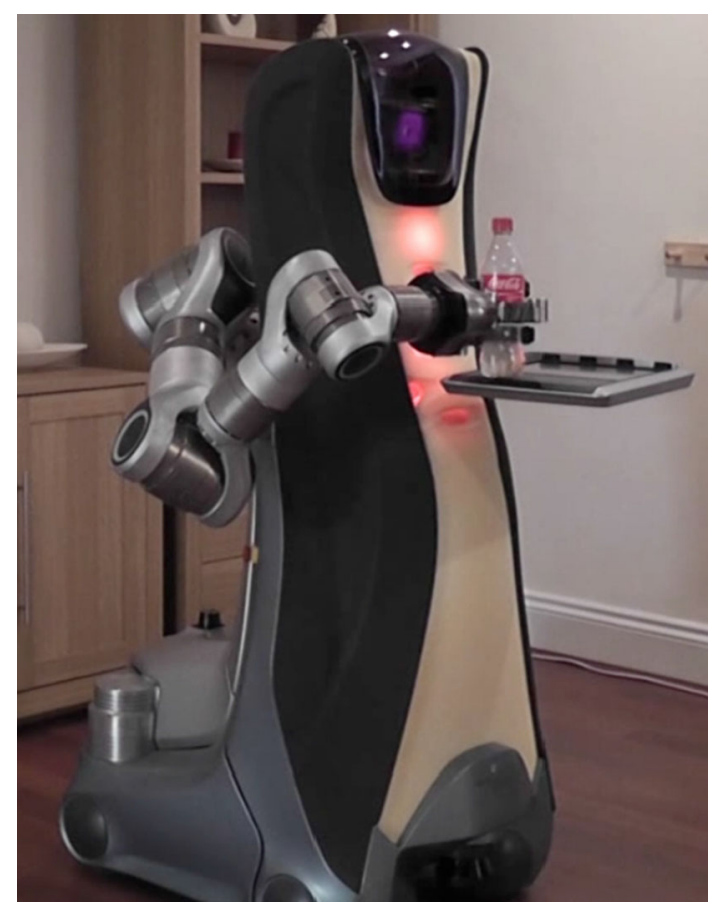

Fig. 5 Care-O-bot ${ }^{\circledR} 3$ placing a bottle on its tray while its LED display panel is showing red colour. (Color figure online) 


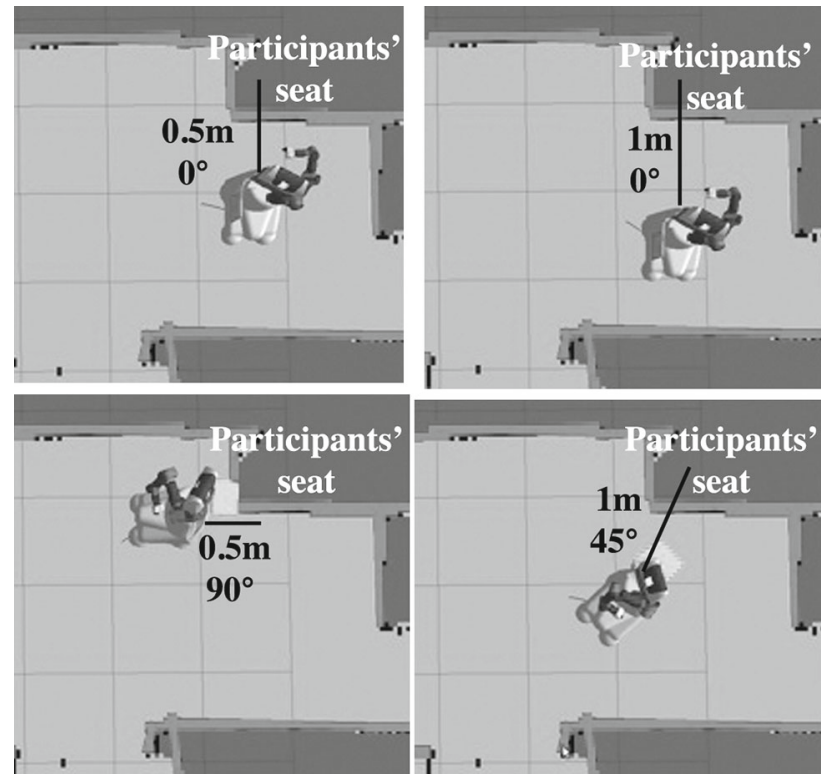

Fig. 6 Care-O-bot ${ }^{\circledR} 3$ stops at the four pre-defined HRP approach positions used in the trial. Clockwise from top is the Front Close, Front Far, Side Far and Side Close HRP poses

is equipped with two SICK laser range scanners (on front and back) and a Hokuyo laser range (top back), tactile sensors on the fingers, a time-of-flight camera and a stereo camera in the head. In this study, the Care-O-bot ${ }^{\circledR} 3$ was used for fetching either a bottle of soft drink or a woolen hat from their designated locations to the participant who was seated on the sofa. The scenario for the experiment assumed that users require physical assistance from the robot, either by serving a drink or handing over clothes required for going out. The Care-O-bot ${ }^{\circledR} 3$ used its manipulator to take an object from the designated location, and then presented it to the participant. If the object was a soft drink bottle, the robot raised its tray, and subsequently placed the bottle on it. If the object was a hat, the robot carried the hat with its hand in front of its chest. It then moved toward one of the four designated pre-defined HRP poses (position and orientation, see Fig. 6 around the participants to present the object to the participant. The Care-O-bot ${ }^{\circledR} 3$ then used speech, (i.e. Here is your clothes for the hat, Here is your drink for the bottle), to invite the participants to take the object. In terms of robot control, the experiment used a combination of Wizard-of-Oz (WoZ, a technique which originated in $\mathrm{HCI}$ but has been used widely in HRI $[16,35])$ remote control and autonomous behaviour, as an experimenter would start each step of the sequence.

\subsubsection{Research Questions}

The study focused on the participants' preferred Care-Obot ${ }^{\circledR} 3$ 's pre-defined HRP poses for presenting the objects to the participants. This was to create a baseline test of preferred HRP poses for the robot in order to evaluate and develop a comfortable experience for the users who interacted with the robot. Also, it was desired to compare HRP preferences from previous studies with robots with different appearances and physical configurations [34]. Two different modes of handing over an object to the user were investigated in order to study if participants' HRP preferences (i.e. how closely and from which direction they preferred the robot to approach) would be influenced by how they were served by the robot.

It was thought that due to the visual appearance of the robots arm and hand, the involvement of the arm and hand may have been perceived to have a higher level of potential hazard compared to being presented with an object transported on its tray. Therefore the hypothesis was proposed:

H1 Participants would prefer the Care-O-bot ${ }^{\circledR} 3$ to stay further away when it was approaching and presenting the hat with its hand, as opposed to presenting the soft drink on its tray.

In addition, as the handing over objects with a gripper requires more concentration, participants may have a greater preference for approaches for this purposes being from the front rather than the side.

H2 Participants would prefer the Care-O-bot ${ }^{\circledR} 3$ to approach from the front when it is presenting the hat with its hand to a larger extent than when it is presenting a soft drink on its tray.

As the light signals were primarily intended to signal movements of base and arm, participants that were cognisant of the signals and their purpose would be more comfortable with the behaviours that they signal. From this, participants recognising the particular colour signalling movement should be more comfortable with close approaches than other participants. In terms of recognizing the colour signalling arm movement, participants who recognise this signal should also be more comfortable with receiving the hat than participants who do not, as this required direct interaction with the arm.

H3a Participants who correctly identify the LED display colour to signal movement will give more positive ratings to closer approaches.

H3b Participants who correctly identify the LED display colour to signal arm movement will give more positive ratings to receiving the hat.

There was also a proposed effect for social role expectations. Handing over a bottle is an interaction that might vary based on the social roles of the interactors. Kendon [27] suggests that positioning based on gaze is important to emphasise the social dimensions of an interaction. Positioning in such a way that mutual gaze is encouraged (such as during a frontal 
approach) makes an interaction between the two interactors more social in nature, while an interaction in which one interactor is outside of the field of vision of the other is much less so. This suggested that high scores on the equality dimension would presuppose a relationship in which the handing over interaction is a social occasion and so participants with a high score on this dimension should prefer that the robot hands over the bottle from the front to a larger extent than those with lower scores on this dimension.

Likewise, the roles in the Control Dimension pre-suppose a more subservient relationship where the robot is more of a servantlike entity, and as such approaches from the side should be preferred to a larger extent for participants with higher scores on this dimension. On the other hand, the handing over of the hat required more coordination and effort between the robot and the participants in terms of movement, and so here, the context would be a stronger influence than the expectations arising from perceived social role.

H4a Scores on the Equality dimension will correlate with positive ratings on the frontal approach for the Bottle Condition.

H4b Scores on the Control Dimension will correlate with positive ratings on the side approach for the Bottle condition.

In terms of long-term expectations, this was an exploratory study for this dimension.

\subsubsection{Experimental Setup}

The overall context was that of a first encounter interaction with the robot (i.e. a guest being served by Care-Obot ${ }^{\circledR} 3$ ). We expected that simple light displays that reflected the robot's current function and mode of operation could be easily identified by the users and thus facilitate HRI. In the study, four HRP presentation poses (i.e. sets of Care-Obot ${ }^{\circledR} 3$ distances and orientations relative to the user) were defined based on results from previous studies [34,60] with PeopleBots focusing on two relatively close positions from the user (i.e. Front Close pose- $-0.5 \mathrm{~m}$ in front of the participants, Side Close pose- $0.5 \mathrm{~m}$ to the right of the participants) and two far positions (i.e. Front Far pose- $1 \mathrm{~m}$ in front of the participants, Side Far $-1 \mathrm{~m}$ and $45^{\circ}$ to the right of the participants) (see Fig. 6).

In terms of human-human proxemics, both of these distances would put the base of the robot within what Hall [18] would refer to as personal space, which is considered appropriate for interaction between friends. However, due to the extension of the tray and the arm, the closer distance would put parts of the robot within what Hall termed intimate space, which is reserved for interactions with close friends and family members. Because of this, the two approach distances were qualitatively different from each other. The HRP poses were used in two different experimental conditions (see below). The task involved the Care-O-bot ${ }^{\circledR} 3$ fetching and presenting different objects to the participants. After the trials, the participants were asked about their preferred HRP presentation poses.

The robot used speech and simple expressive behaviours (i.e. different colour LED light signals) to provide feedback to the user as described previously. The different colour LED lights in the robot's chest essentially displayed an Interaction Alert Level. The Interaction Alert Level corresponded to the potential level of hazard present in the task or actions the robot was currently executing, in order to facilitate safe interaction between the user and the robot. The Care-O-bot ${ }^{\circledR} 3$ displays a steady white colour when its ready/safe for interaction, a blinking yellow colour to signal to the user to be cautious around the Care-O-bot ${ }^{\circledR} 3$ when it is moving or navigating (i.e. the robot is capable of avoiding both static and moving obstacle, but a user should be cautious as they might coincidently reverse into the robot), and a blinking red colour when it is moving its arm (i.e. when user is in the vicinity of the robot they should pay attention to the robot).

The purpose of this expressive channel was not revealed to the participants during the trial in order to see if participants could intuitively derive the meaning of the robot's coloured LED light signals. The participants completed a questionnaire which gained their preferences and perceptions of the pre-defined HRP poses and their reasoning behind their choices. Also, their perception of the robot's colour LED light signals was elicited.

\subsubsection{Experimental Procedure}

Two experimenters were involved in the trial. A psychologist introduced and explained the trial procedure, handed out questionnaires to the participants and answered any questions participants might have about the trial. There was also a roboticist present who monitored the robot to ensure it executed its tasks as planned, and monitored safety for the participants (via a wireless emergency stop button for the robot). The trial was supervised by at least one experimenter at all times. Figure 7 shows examples of a participant collecting objects from Care-O-bot ${ }^{\circledR} 3$ at four different pre-defined HRP poses. The experimental procedure was as follows:

Introduction First a welcome phase where participants were introduced to the UH Robot House and the Care-Obot ${ }^{\circledR} 3$. They signed a consent form and completed a demographics questionnaire and a questionnaire regarding social roles. They were then shown a live demonstration of the robot autonomously executing its tasks which was based on Condition Bottle (see below). The participants were free to move around to see how the robot performed its tasks. The demon- 


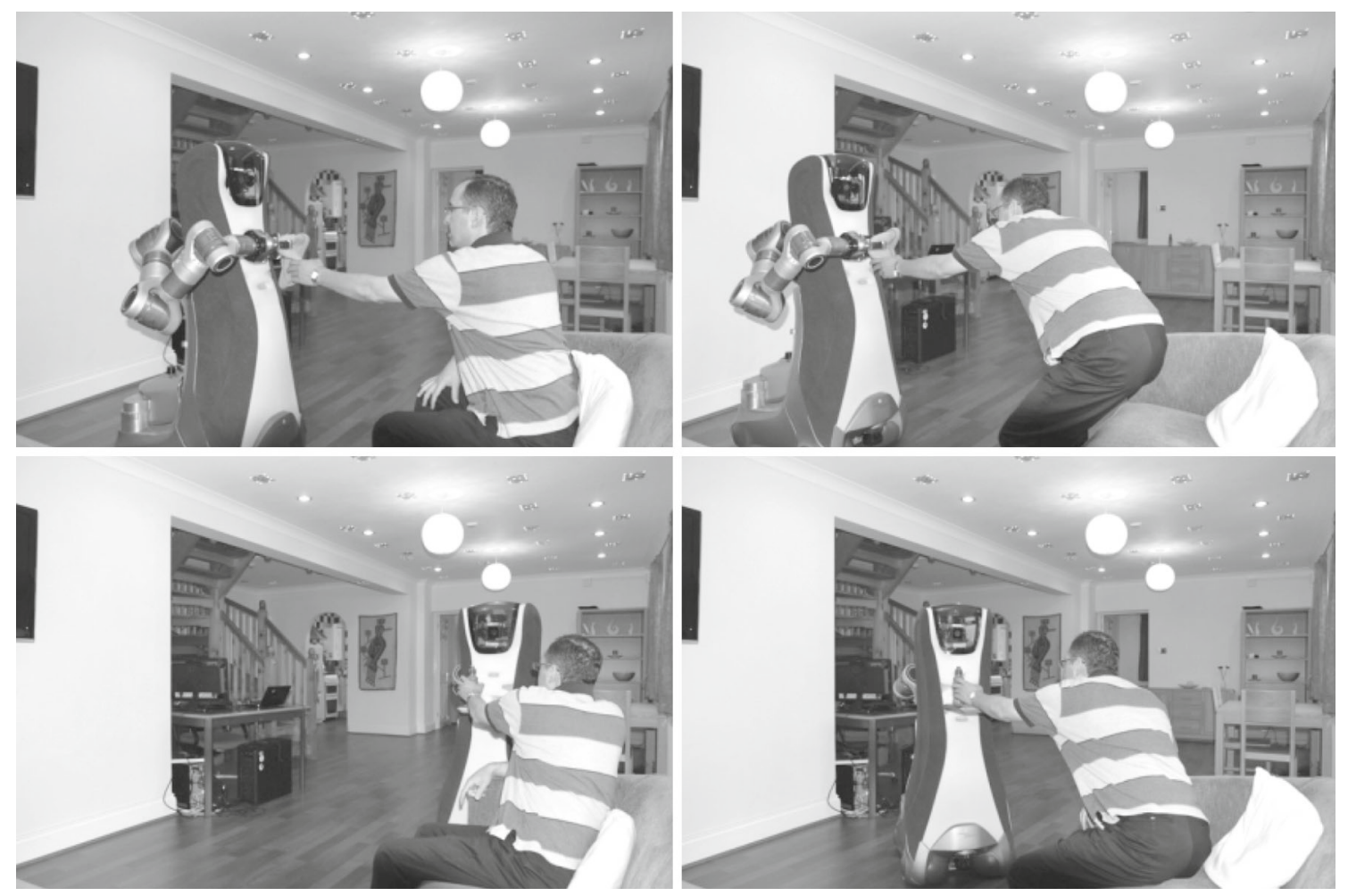

Fig. 7 An example of a left-handed participant fetching an object from Care-O-bot ${ }^{\circledR}$ 3's tray/hand at the four pre-defined HRP approach positions used in the trial. Clockwise from top is the Front Close, Front Far, Side Far and Side Close HRP poses

stration was to settle any initial curiosity the participants may have regarding how the robot might perform fetching and presenting objects during the trial.

Main Trial The main trial consisted of two different conditions of Care-O-bot ${ }^{\circledR} 3$ fetching and presenting an object to the participants. The conditions were differentiated by the object it was fetching and presenting to the participants. The order of approach direction and distance was randomized within each condition. In addition, the order of conditions was also randomized, however, a participant would experience all approaches for each condition before being exposed to the next condition.

Condition Bottle Involved the robot leaving its station to fetch a bottle of soft drink from a shelf, lift its tray, place the bottle on its tray, park its arm, move to one of the pre-defined HRP points and present the bottle, using speech (i.e. Here is your drink) to invite the participants to take it. After the bottle was taken, the robot moved back, lowered its tray and returned to its station.

Condition Hat Involved the robot leaving its station to fetch a hat from the clotheshanger, move the hat with its hand positioned to its front end in front of its chest, move to one of the pre-defined HRP poses, then presented the hat, using speech (i.e. Here is your clothes") to invite the participants to take it. After the hat was taken, the robot moved back, parked its arm and returned to its station.
In both conditions, the participants were asked to sit at a designated location on the sofa. Each condition was repeated four times, each with the robot ending its approach at a different pre-defined HRP pose around the user. At the end of the fourth repeat of each condition, participants were then asked to complete a second questionnaire regarding their experiences before they proceeded to experience the other condition. Final Questionnaire: Participants were then asked to complete a final questionnaire, which asked them to recall the colour displayed by the robot's LED display when it moved its arm and when it moved around the room and why the colours were different.

\subsubsection{Measures}

Participants' social role expectations of the robot were assessed in a pen and paper questionnaire using the same questions as described in Sect. 2.1. As in the survey study, the term robot was not more closely defined, although the participants had seen the Care-O-bot ${ }^{\circledR} 3$ at this point. Participant responses to the robot's approaches were assessed using a questionnaire. An ad-hoc questionnaire was created for this study, building on previous research carried out at the UH Robot House. These findings suggest that while responses to proxemic behaviour are often discussed in terms of practicality, there are also other factors such as comfort or 
Table 5 Questionnaire items used in the study

\begin{tabular}{ll}
\hline Item & Factor \\
\hline $\begin{array}{l}\text { It made more sense for giving me this object than } \\
\text { some of the other approaches }\end{array}$ & Practicality \\
$\begin{array}{l}\text { It was intimidating compared to some of the } \\
\text { other approaches }\end{array}$ & Hedonic \\
$\begin{array}{l}\text { It was less practical for taking the object than } \\
\text { some of the other approaches }\end{array}$ & Practicality \\
$\begin{array}{l}\text { It made me feel more comfortable than some of } \\
\text { the other approaches }\end{array}$ & Hedonic \\
\hline
\end{tabular}

feelings of threat, that may impact how a participant evaluates an interaction. We refer to these factors as hedonic factors $[34,48]$. Due to the large number of approaches, brevity was a major concern in the questionnaire design, with four items being considered the highest number that participants could be expected to complete per approach. The items are presented in Table 5 and participants were asked to rate these items in term of agreement (i.e. 1: Completely Disagree, 2: Disagree, 3: Neutral, 4: Agree, 5: Completely Agree). After each condition run, participants were also asked to choose which approach was the most comfortable and practical. At the end of the trial, a series of open-ended questions were used to assess participants recall and comprehension of the LED signaling.

\subsubsection{Participants}

The participants for these two studies (i.e. both long- and short-term sample) were recruited through advertisements on UH mailing lists and the University Intranet.

The short-term sample consisted of participants that were freshly recruited for this study. These participants had never visited the UH Robot House nor seen a real Care-O-bot ${ }^{\circledR} 3$ prior to the study. Therefore their experiences may be equivalent to a first encounter situation, such as that of a new user or a guest being served by Care-O-bot ${ }^{\circledR} 3$. There were 19 participants in the short-term study (i.e. 12 male and 7 female participants). The mean age for the participants were 26 with a median age of 22.5. Seventy percent of the participants were between the ages of 19 and 25. Nine participants had experience of computer programming, while the other 10 did not. The short-term participants only interacted with the Care-Obot ${ }^{\circledR} 3$ once within the experimental setting. This sample was used to investigate the issue of baselines and preferences at an initial meeting (first encounter).

The long-term sample had just completed the first nine weeks of a long-term robot companion HRI study, which consisted of one hour session per week, with a total of 9 sessions. In this long-term study, participants mainly interacted with a different robot that provided physical (carrying object, a similar task to the one in this trial) and cognitive assistance (reminders) around the UH Robot House on day-to-day activ- ities. Therefore this sample of participants were very familiar with the UH Robot House and the Care-O-bot ${ }^{\circledR} 3$ had already been encountered in static mode prior to the current study as it was at its charging station in the same room as the long-term study was being conducted in. The long-term sample had 11 participants, 4 female and 7 male. The mean age for the participants was 32 , with a median age of 26 . Fifty percent were between 19 and 25. The long-term participants were used to interacting with autonomous, mobile robots in a variety of tasks. This sample responded to the question regarding the preferred social roles for the robot in the first week of the long-term study. This sample, due to their greater experience of robots moving autonomously in the Robot House setting, were used to investigate how a baseline may differ for participants with more HRI experience.

\section{Results}

\subsection{Reliability Analysis}

The reliability of the measures were assessed through a series of Reliability tests with each measure assessed for each condition. The mean Cronbach's $\alpha$ was .79 for the Practicality measure, and .52 for the Hedonic measure. The high score for Practicality is particularly encouraging, although the low sample size of this study means that we can only tentatively consider these items a reliable measure. The lower score for the Hedonic measures is more problematic, but due to the high face validity of these items, it was decided to consider them as a single measurement for this study. However, results arising from these will have to be considered in the light of their low reliability.

\subsection{Short-term Sample}

\subsubsection{Ratings}

Figure 8 and Table 6 show the descriptive statistics for the Practicality ratings of the short-term sample. There were no significant main effects for Object' $[F(1,17)=.290, p=$ $\left..60, \eta^{2}=.02\right]$ or Direction $[F(1,17)=1.076, p=$ $\left..314, \eta^{2}=.06\right]$. There was, however a significant effect for Distance $\left[F(1,17)=43.053, p<.001, \eta^{2}=.72\right]$, suggesting that participants viewed close approaches as more practical overall (Mean Rating of Closer was 4.12, SE .13, Mean rating for Further was 3.19, SE .17). There were no significant interactions.

Table 7 shows the descriptive statistics for the Hedonic ratings of the short-term sample. There were no significant main effects for Object $\left[F(1,17)=.004, p=.95, \eta^{2}<.001\right]$ for Distance $\left[F(1,17)=1.563, p=.23, \eta^{2}=.08\right]$. There was, however, an effect approaching significance for 


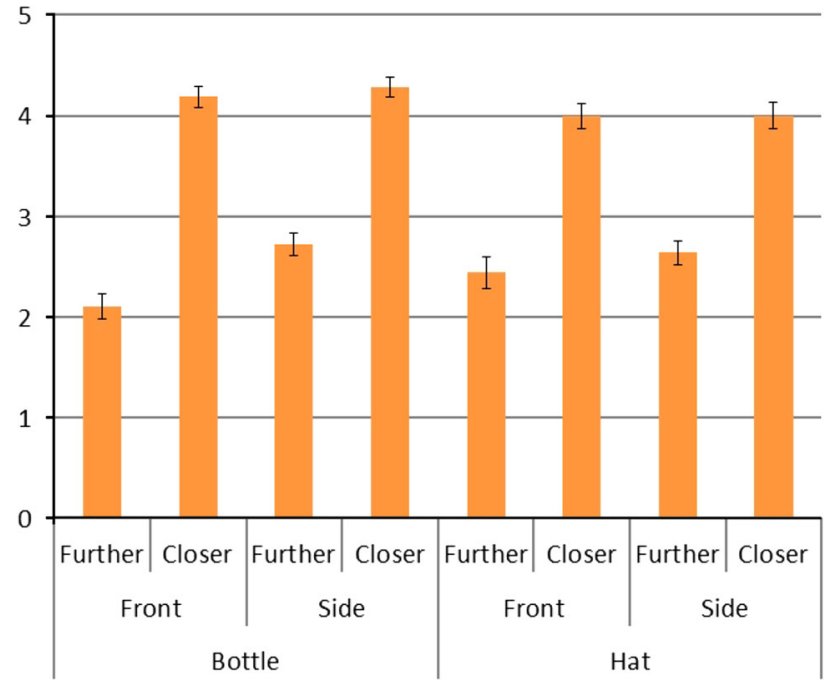

Fig. 8 Practicality ratings for the short-term sample

Table 6 Practicality ratings for the short-term sample

\begin{tabular}{lllll}
\hline Object & Direction & Distance & Mean rating & SE \\
\hline Bottle & Front & Further & 2.11 & 0.25 \\
& & Closer & 4.19 & 0.21 \\
& Side & Further & 2.72 & 0.23 \\
& & Closer & 4.28 & 0.20 \\
Hat & \multirow{2}{*}{ Front } & Further & 2.44 & 0.32 \\
& & Closer & 4.00 & 0.25 \\
& \multirow{2}{*}{ Side } & Further & 2.64 & 0.24 \\
& & Closer & 4.00 & 0.27 \\
\hline
\end{tabular}

Table 7 Hedonic ratings for the short-term sample

\begin{tabular}{lllll}
\hline Object & Direction & Distance & Mean rating & SE \\
\hline Bottle & Front & Further & 2.92 & 0.21 \\
& & Closer & 3.31 & 0.23 \\
& \multirow{2}{*}{ Side } & Further & 3.33 & 0.20 \\
& & Closer & 4.14 & 0.18 \\
Hat & \multirow{2}{*}{ Front } & Further & 3.36 & 0.17 \\
& & Closer & 3.31 & 0.19 \\
& \multirow{2}{*}{ Side } & Further & 3.50 & 0.20 \\
& & Closer & 3.50 & 0.20 \\
\hline
\end{tabular}

Direction $\left[F(1,17)=3.277, p=.09, \eta^{2}=.16\right]$. This effect suggested that participants rated the Side approaches more favourably in terms of Hedonic qualities (Mean score for Front Approaches 3.22, SE .12, Mean Score for Side Approaches 3.62, SE .18). There was a significant interaction effect for Object and Direction. This interaction effect is described in Table 8 and Fig. 9 which suggests that it is the ratings for the Bottle that are responsible for the preference for side approaches, while for the Hat, there is less impact of
Table 8 Interaction effect for Hedonic ratings for the short-term sample

\begin{tabular}{llll}
\hline Object & Direction & Mean rating & SE \\
\hline Bottle & Front & 3.11 & 0.17 \\
& Side & 3.74 & 0.16 \\
\multirow{2}{*}{ Hat } & Front & 3.33 & 0.12 \\
& Side & 3.50 & 0.16 \\
\hline
\end{tabular}

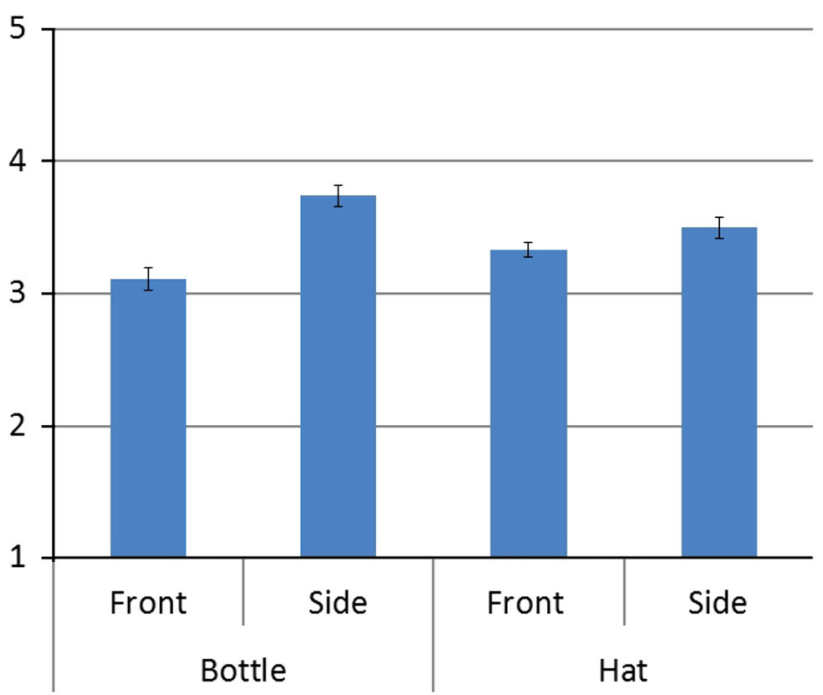

Fig. 9 Interaction effect for Hedonic ratings for the short-term sample

direction. These results are not in support of hypothesis $\mathrm{H} 1$ as there is no difference in either Practically ratings or Hedonic ratings for the Bottle or Hat in terms of distance. This could be due to the closer approaches being overall viewed highly favourably, which may have masked any effect of object. The results presented here can be taken to provide some support to hypothesis $\mathrm{H} 2$ as there is a trend for participants to view Frontal approaches for the Hat more favourably in terms of practicality than those for the Bottle, and the Hedonic ratings in which participants only distinguished between the two directions for the bottle but not for the hat. As for the Hedonic ratings reported in Table 8, this offers support to Hypothesis $\mathrm{H} 2$.

\subsubsection{Preferences}

In terms of which particular approach was the most practical and comfortable, the results from the Bottle condition presented in Table 9 shows significant deviations from an equal distribution for both Practicality (Fisher's Exact $p<.001$ ) and Comfort (Fisher's Exact $p<.001$ ). A subsequent examination of the residuals suggest that the far approaches are the least preferred, while the closer ones, in particular from the side, are the most preferred.

The results for the Hat condition approaches presented in Table 10, show there were significant deviations from an 
Table 9 Preferences in terms of practicality and comfort for the bottle condition for the short-term sample

\begin{tabular}{lcccc}
\hline Direction & Practicality & Residual & Comfort & Residual \\
\hline Front Far & 0 & -4.8 & 0 & -4.8 \\
Front Near & 4 & -0.8 & 4 & -0.8 \\
Side Far & 0 & -4.8 & 1 & -3.8 \\
Side Near & 15 & 10.3 & 14 & 9.3 \\
\hline
\end{tabular}

Table 10 Preferences in terms of practicality and comfort for the hat condition for the short-term sample

\begin{tabular}{lcclc}
\hline Direction & Practicality & Residual & Comfort & Residual \\
\hline Front Far & 0 & -4.8 & 0 & -4.8 \\
Front Near & 3 & -1.8 & 9 & 4.3 \\
Far & 3 & -1.8 & 3 & -1.8 \\
Side Near & 13 & 8.3 & 7 & 2.3 \\
\hline
\end{tabular}

equal distribution for both Practicality (Fisher's Exact $p<$ .001 ) and Comfort (Fisher's Exact $p<.001$ ). A subsequent examination of the residuals suggests a similar result as for the Bottle condition approaches in terms of practicality, but not in terms of Comfort. Here there is no real difference between the side and the front approaches.

\subsubsection{LED Light Display}

Participants did not correctly remember the colours of the LED light display for the different behaviours more than could be expected due to chance $\left[\chi^{2}(1)=.22, p=.637\right]$. The majority of participants did however, correctly identify the intended function of these lights as one of alerting the participant to the robot's behaviour $\left[\chi^{2}(1)=5.56, p=.02\right.$ (14 Correct, 4 Incorrect, and 1 did not answer the question)]. This suggests that over time, participants would potentially be able to utilize such a system to identify the robot's intentions, and that possibly the novelty of the interaction scenario made it more difficult to retain this information. Moreover, the ability to correctly identify the LED display signal colour when the arm was moving, interacted with overall ratings of object type $\left[F(1,15)=4.51, p=.046, \eta^{2}=.23\right]$. This effect is shown in Table 11 and Fig. 10, which show that participants that did not identify the colour used, differentiated between the bottle and the hat, in their ratings, while participants who did correctly identify them, did not. This suggests that the use of the lights had an impact in how the participants perceived the manner in which the object was handed over to them.

These results mean that we cannot accept hypothesis H3a. However, they can be taken as limited support for hypothesis $\mathrm{H} 3 \mathrm{~b}$, as participants that correctly identified the colour used
Table 11 Interaction effect of participants who correctly identified the colour of the LED light display when the arm is moving and the object type

\begin{tabular}{llll}
\hline Correctly identified & Object & Mean rating & SE \\
\hline Yes & Bottle & 3.06 & 0.11 \\
& Hat & 3.18 & 0.10 \\
No & Bottle & 3.46 & 0.13 \\
& Hat & 3.18 & 0.12 \\
\hline
\end{tabular}

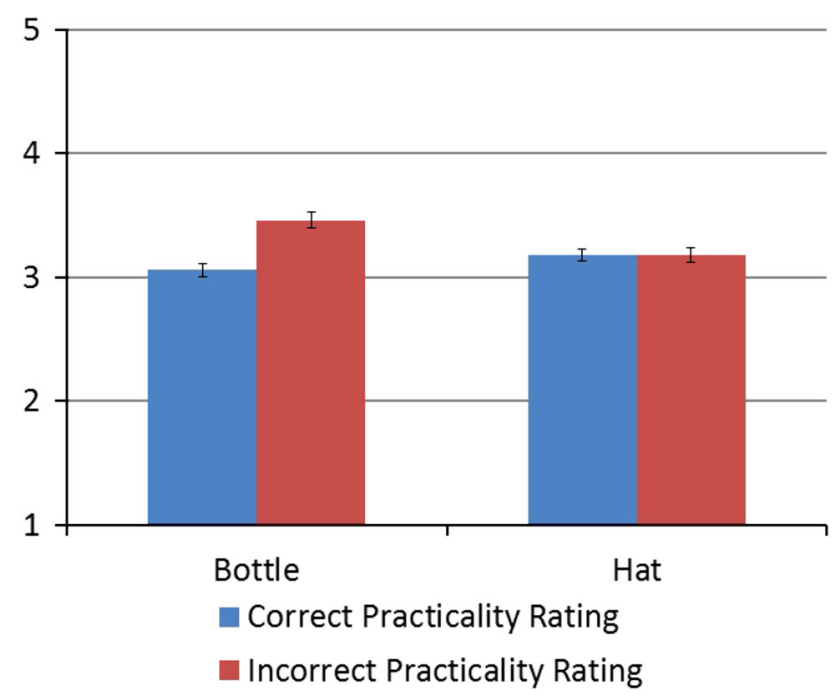

Fig. 10 Interaction effect for correctly identifying the colour of the LED light display when the arm is moving and the practicality ratings for object type

Table 12 Correlations for equal social role expectations and practicality ratings for approach direction

\begin{tabular}{llll}
\hline Variable & Equal & Frontal approach & Side approach \\
\hline Equal & 1 & & \\
Frontal approach & $.584 *$ & 1 & \\
Side approach & .110 & .202 & 1 \\
\hline$p<.05$ & & &
\end{tabular}

for moving the arm did not differentiate between the bottle and arm conditions, while those who did not, rated the bottle condition more favourably.

\subsubsection{Social Role Expectations}

Proxemics The relationship between social role expectations and proxemics were assessed using a series of Spearman correlations. In terms of proxemics, the results described in Table 12 support Hypothesis 4a in that participants scoring higher on the Equality dimension in terms of social role expectations, were more likely to rate the Frontal Approaches more favourably in terms of Practicality than those scoring lower on this dimension. 
Table 13 Correlations for control social role expectations and practicality ratings for approach direction

\begin{tabular}{lccc}
\hline Variable & Control & Frontal approach & Side approach \\
\hline $\begin{array}{l}\text { Control } \\
\text { Frontal approach }\end{array}$ & 1 & & \\
Side approach & $.462^{*}$ & .202 & 1 \\
\hline$* p<.05$ & & & \\
Table 14 Control dimension and LED display identification
\end{tabular}

Likewise, Hypothesis $4 \mathrm{~b}$ was also supported by the results. Table 13 suggests that participants scoring higher on the Control Dimensions were more likely to rate the Side Approaches more favourably in terms of practicality as well.

There were no significant correlations between the scores on the social role expectations and the Hedonic ratings of the approach direction, suggesting that proxemic preferences were considered more in terms of sense and practicality than emotional response. There were also no significant relationship between scores on the dimensions of social role expectations and preferences for the Hat condition, supporting the notion that the coordination requirements of the task investigated in Hypothesis 2 superseded the expectations arising from the social role expected of the robot.

LED Light Display There was a significant effect for the Control Dimension and correctly remembering the colours of the LED Light Display for the different behaviours. This effect is shown in Table 14 and Fig. 11, which suggests that participants that correctly remembered the colours used, scored significantly lower on this dimension than participants who did not.

\subsection{Long-term Sample}

\subsubsection{Ratings}

Table 15 shows the descriptive statistics for Sample B's Practicality ratings. There were no significant main effects for Object $\left[F(1,10)=.190, p=.67, \eta^{2}=.02\right]$ nor was there one for Direction $\left[F(1,10)=.172, p=.68, \eta^{2}=.02\right]$. There was a main effect that was approaching significance

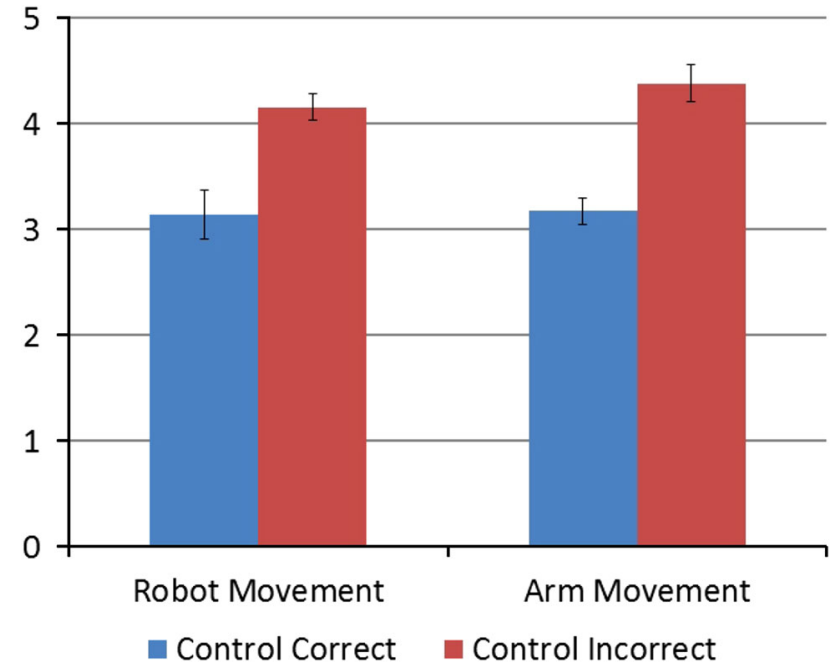

Fig. 11 Control dimension and LED display identification

Table 15 Practicality ratings for the long-term sample

\begin{tabular}{lllll}
\hline Object & Direction & Distance & Mean rating & SE \\
\hline Bottle & Front & Further & 3.05 & 0.11 \\
& & Closer & 3.18 & 0.15 \\
& Side & Further & 2.82 & 0.10 \\
& & Closer & 3.32 & 0.17 \\
Hat & Front & Further & 2.82 & 0.14 \\
& & Closer & 3.14 & 0.24 \\
& Side & Further & 3.05 & 0.08 \\
& & Closer & 3.18 & 0.15 \\
\hline
\end{tabular}

for Distance $\left[F(1,10)=3.161, p=.11, \eta^{2}=.24\right]$. This effect is described in Fig. 12 and suggests that participants rated the closer approach distances as more practical than the approaches to the further distances (Mean for Closer is 3.20 with an SE of .06, and the Mean for Further is 2.93 with a $\mathrm{SE}$ of .11). There were no significant interaction effects for Practicality ratings.

Table 16 and Fig. 13 shows the descriptive statistics for the Hedonic ratings for the long-term sample. There was an effect approaching significance for Object $[F(1.10)=$ $\left.4.925, p=.051, \eta^{2}=.33\right]$. This effect suggested that participants rated the handing over of the hat more favourably than the bottle (Mean for the hat was 2.88 with an SE of .09 , and the Mean for the bottle was 2.72 with a SE of .11). There was a significant main effect for Distance $\left[F(1,10)=6.397, p=.03, \eta^{2}=.39\right]$. This effect suggested that the closer distances were rated more favourably than the further distances in terms of Hedonic qualities (Mean for the Closer was 3.00 with an SE of .08. The Mean for Further was 2.59 with a SE of .16). There was no significant main effect for Direction $\left[F(1,10)=2.642, p=.14, \eta^{2}=.21\right]$ and no significant interaction effects for the Hedonic ratings. 


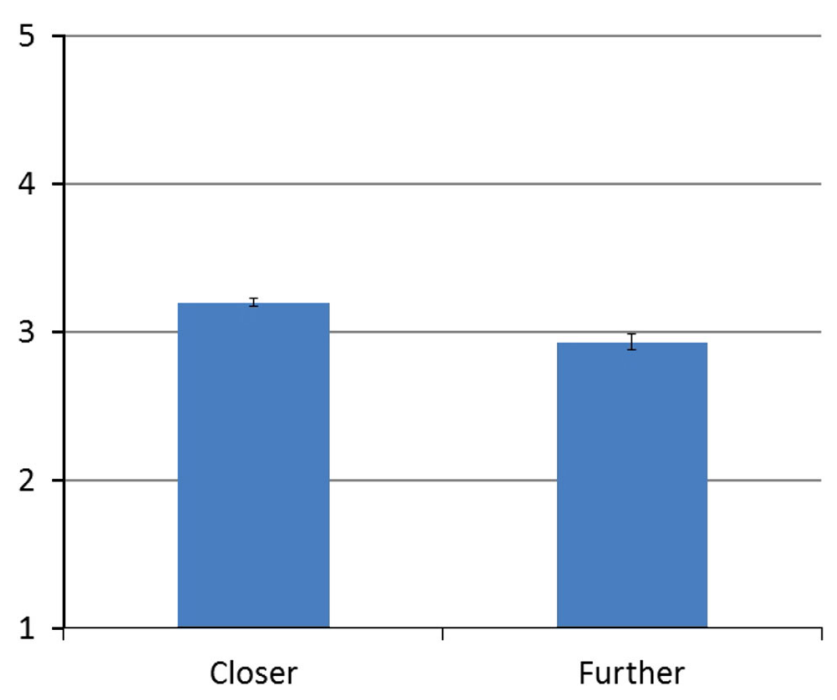

Fig. 12 Practicality ratings by distance long-term sample

Table 16 Hedonic ratings for the long-term sample

\begin{tabular}{lllll}
\hline Object & Direction & Distance & Mean rating & SE \\
\hline Bottle & Front & Further & 2.50 & 0.23 \\
& & Closer & 2.77 & 0.19 \\
& Side & Further & 2.50 & 0.18 \\
& & Closer & 3.09 & 0.09 \\
Hat & \multirow{2}{*}{ Front } & Further & 2.45 & 0.17 \\
& & Closer & 3.00 & 0.17 \\
& \multirow{2}{*}{ Side } & Further & 2.91 & 0.21 \\
& & Closer & 3.14 & 0.07 \\
\hline
\end{tabular}

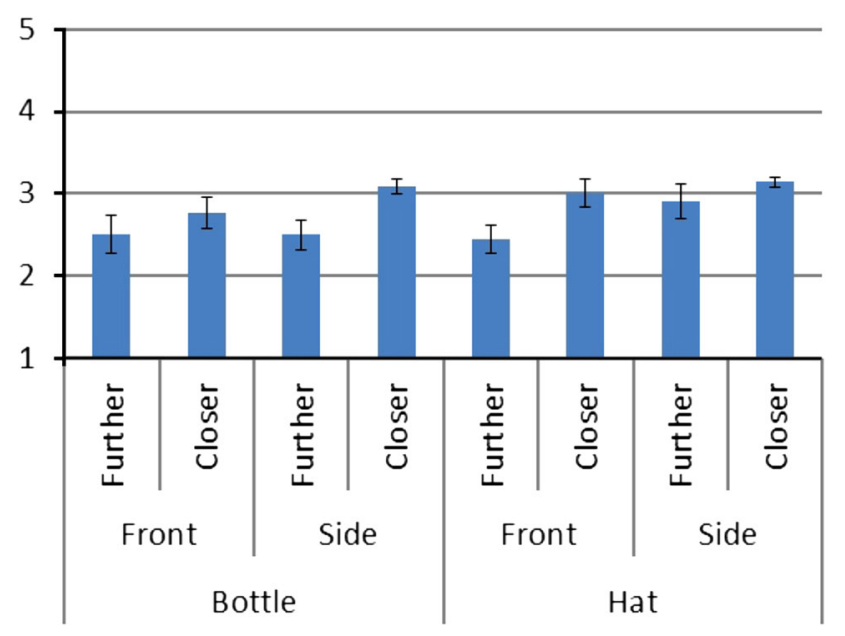

Fig. 13 Hedonic ratings for the long-term sample

\subsubsection{Preferences}

In terms of which particular approach was the most practical and comfortable, the results from the Bottle condition approaches presented in Table 17 shows deviations from an
Table 17 Preferences in terms of practicality and comfort for the bottle condition for the long-term sample

\begin{tabular}{llclc}
\hline Direction & Practicality & Residual & Comfort & Residual \\
\hline Front Far & 0 & -2.8 & 0 & -2.8 \\
Front Near & 2 & -0.8 & 2 & -0.8 \\
Side Far & 3 & 0.3 & 2 & -0.8 \\
Side Near & 6 & 3.3 & 7 & 4.3 \\
\hline
\end{tabular}

Table 18 Preferences in terms of practicality and comfort for the hat condition for the long-term sample

\begin{tabular}{llclc}
\hline Direction & Practicality & Residual & Comfort & Residual \\
\hline Front Far & 0 & -2.8 & 1 & -1.8 \\
Front Near & 2 & -0.8 & 0 & -0.8 \\
Side Far & 1 & -1.8 & 2 & -0.8 \\
Side Near & 8 & 5.3 & 7 & 4.3 \\
\hline
\end{tabular}

equal distribution approaching significance for Practicality (Fisher's Exact $p=.079$ ) and a significant deviation for Comfort (Fisher's Exact $p=.020$ ). The residuals suggest that for both Practicality and Comfort, the side approaches were preferred to a greater degree than the front approaches, and closer approaches to further approaches.

The results from the Hat condition's approaches are presented in Table 18. There were significant deviations from an equal distribution for both Practicality (Fisher's Exact $p=.004$ ) and Comfort (Fisher's Exact $p=.004$ ). The residuals suggest that the side near approaches were the most preferred in terms of practicality and comfort.

Table 18 Preferences in terms of Practicality and Comfort for the Hat condition. Direction Practical Residual Comfortable Residual.

\subsubsection{LED light display}

In the long-term study, participants did not correctly remember the colours of the LED light displays more than could be expected from chance $\left[\chi^{2}(1)=2.73, p=.14\right]$. Likewise, participants did not guess the function of the colours more than could be expected through chance $\left[\chi^{2}(1)=2.73, p=\right.$ .14]. There was no impact of having guessed correctly on either Practicality or Hedonic Ratings.

\subsubsection{Social Role Expectations}

There were no significant correlations between Social Role Expectations and the proxemic preferences in the Care-Obot ${ }^{\circledR} 3$ Study for the long-term sample. Likewise, there was no relationship between the Social Role Expectations and remembering the colours used to signal the different behav- 
iours. This is in line with Ljungblad et al. [39]s view of mental models in terms of social roles as changing over time.

\section{Analysis of Findings}

\subsection{Hypothesis H1: Object and Distance}

The findings from both the short- and long-term sample did not support hypothesis H1. Participants did not distinguish between the two objects in terms of what distance they wanted the robot to approach to. This could be due to the high preference in the sample for the closer distance, despite this distance being within what Hall [18] termed intimate space, which might have created a ceiling effect for the data. This ceiling effect might have been caused by the difference in perceived effort experienced by the participants, as the further distance would require them to reach out longer with their arms or even stand up to pick up the objects from the robot. Other possibilities include that the participants did not consider the arm more threatening than the tray; or that the direct interaction of having to coordinate ones behaviour with the robot in this particular scenario was richer and more rewarding. The results in terms of preferences of objects in the long-term, suggest that this might be the case. This group, which already had interacted with robots and would therefore find this interaction less novel, rated this interaction more favourably in terms of Hedonic ratings. This is likely the results of a similar habituation effect as that reported in Koay et al. [34].

\subsection{Hypothesis H2: Object and Direction}

The findings from the short-term sample lent support to hypothesis H2. Participants did distinguish between the objects in terms of how they rated the directions from which they were presented them. This suggests that the coordination between robot and human required for the handing over of the hat led to a greater preference for the frontal approach than for the bottle. This finding was not replicated in the longterm sample. This can suggest that the mental requirements of coordination were primarily an issue when participants were interacting with a novel robot.

\subsection{Hypothesis H3: Signalling}

The participants responses to the questions regarding lights suggested that participants struggled with recalling the light colours used to signal the different behaviours of the robot. $\mathrm{H} 3 \mathrm{a}$ regarding understanding movement was not supported by the data, similarly as for $\mathrm{H} 1$, and this could be the result of a ceiling effect. An alternative explanation might be that the movement of the robot was clear enough to not need disam- biguation by the LED signals. H3b, on the other hand, was somewhat supported by the data. Participants who did not identify the colour used to signal arm movement, preferred the bottle over the hat, while participants who did identify it correctly did not prefer one over the other. This suggests that the signalling used may have had the desired impact for this object. However, an alternative explanation may have been that participants who found the human-robot coordination aspect of this task rewarding may also have been predisposed to spot these signals.

\subsection{Hypothesis H4: Social Roles}

Both Hypotheses $\mathrm{H} 4 \mathrm{a}$ and $\mathrm{H} 4 \mathrm{~b}$ were supported to some extent by the data. Participants did rate the approach directions more congruent with the social role dimensions, as more practical for the bottle condition. This suggests that the social role that the participants expect the robot to have, impacted on how they perceive the context. As suggested earlier, the increased effort involved in coordination between human and robot suggested by $\mathrm{H} 2$ may be the reason this effect did not occur when participants were given the hat.

In addition, the results from Sect. 3.2.4 suggest that participants' expectations in terms of social roles also had an impact on how participants perceived the LED signal displays. Participants who correctly identified the signals tended to score lower on the control dimension than those who did not. A likely explanation for this is that participants with lower scores on this dimension would be more likely to consider the robot as an autonomous agent and be more alert to its signals.

\subsection{Differences Between the Samples}

Overall, analysis of the results from both the short and longterm sample ratings seem to suggest that as people's experiences with robots increase, so do their level of comfort being around the robot. This effect is demonstrated by both samples rating close approach distances as more practical for handing over objects, but only the long-terms sample rated the closer approaches more favourably in terms of the Hedonic dimension. Similarly, participants from the long-term sample, who were already accustomed to a different robot that provided similar functionality, did not differentiate between the approach directions of the robot.

\section{Discussion and Conclusions}

These results were obtained using the Care-O-bot ${ }^{\circledR} 3$ and based on the four pre-defined HRP points (taking into account the robot's position and orientation near the user), as well as robot light signaling. Implications of this work are as follows: 
Firstly, the results allow for the production of initial HRP guidelines for human-robot spatial relationships to help the development of a human aware navigation algorithm [48,52] for the Care-O-bot ${ }^{\circledR} 3$. As mentioned in Sect. 1.3, this is important due to the unique design of the Care-O-bot ${ }^{\circledR} 3$, in particular the size and weight of the robot, as well as its two distinct sides (presentation side with screen and working side with industrial robot arm). The findings from this study will inform the development of the Care-O-bot ${ }^{\circledR} 3$ with regard to how it can perform fetch and carry tasks with both manipulator and tray in a socially appropriate manner.

The preferences from the short-term sample suggest a baseline in which the Care-O-bot ${ }^{\circledR} 3$, when presenting an object on its tray to most users or their guests, should try to approach from the side at a position about $0.5 \mathrm{~m}$ from the users. If the users Social Role Expectations for the robot are high on the Equality dimension, or they happen to be in a situation where it cannot approach from the side, a frontal approach is a possible option. When presenting an object with its gripper, the Care-O-bot ${ }^{\circledR} 3$ should endeavour to approach in the user's field of vision, particularly if this user does not have much experience with robots (e.g. a guest in the household). While these baselines were obtained using a seated participant, they can be built upon to explore other situations [60]. In addition, we can also use them for exploring habituation effects as the ones we observed previously in [34].

We expected that participants would become more comfortable with the robot over time and contrasting the results in terms of Practicality and Hedonic ratings between the shortterm and the long-term samples suggests that is indeed the case. The long-term sample practicality ratings tend to have lower Standard Errors suggesting less variance in the scores, and thus greater agreement within this sample. In addition, apriori Social Role expectations of robots become less important as the participants mental models of the robot become more like its actual capabilities. Further studies need to be conducted to verify if these HRP results are applicable to other contexts where the Care-O-bot ${ }^{\circledR} 3$ is required to interact with participants.

Secondly, the results from using the LED display signals are encouraging. Identifying the signal for arm movement impacted how participants rated the different conditions relative to each other. This lends credence to the notion of using explicit signals to disambiguate the intentions of the robot. However, the results with regards to the Social Role expectations, suggest that the robot may need to present itself more strongly as an autonomous entity in order for users to actively utilize them. This will need to be studied in future investigations by attempting to actively elicit particular expectations in a similar manner to that suggested by Powers et al. [43]. These findings could be generalised across different robotic platforms with similar appearance. For the long-term sample, results are less clear and this may be due to the participants from this sample already having adapted to a different LED light display modality which was used on the other robot in the long term study prior to interacting with the Care-Obot ${ }^{\circledR} 3$. In that study, the robot used the LED light display to express intentionality such as attracting the participants' attention. In addition, the users could personalise this behaviour. Also, the long-term sample were likely to be overall more comfortable with the robot. Therefore a ceiling effect may have masked any impact from correctly identifying the lights used to signal behaviours. This particular issue suggests a more detailed examination of the effect of signals such as LED light displays for experienced users in the future. This could be extended to examine the use of the LED light display as a simple feedback mechanism for mediating turntaking between users and the robot. This simple signalling method would make interactions between users and robot safer. It could also reduce the cognitive load on users when accepting new technologies into their environment and its use could be extended to domains other than proxemics.

A similar result obtained in this study with regards to direction of approach was also found in our previous studies using PeopleBot robots [65]. This suggests that the role of the lights in signalling robot intention can be applied to other robotic platforms. As such, they contribute to our growing understanding of HRP and HRI.

With this baseline HRP information, we are now better equipped to further our HRI studies using the Care-O-bot ${ }^{\circledR} 3$ as a robotic butler, tool or companion that can offer physical assistance and cognitive prosthetic in a comfortable way for the users. The findings here also demonstrate that a study such as this can provide useful baseline information that is vital for implementing user-friendly robots. Future work should also focus on creating a baseline study protocol that can help extract important fundamental HRP factors, so that standardised baseline studies may be replicated with other robots in similar interaction contexts, tasks or user groups, drawing on measurements of aspects not necessarily dependent on the robot platform itself. These might include measurements of participant expectations and other idiosyncratic factors to increase the validity and cross-study value of the studies.

This study highlights that how a robot presents itself, or is presented by others, to the user, significantly impacts preferences in terms of proxemics, as well as the users ability to correctly process its signals in early interactions. This research aims at building a body of knowledge that can inform future HRI studies in a systematic manner. New robotic systems and designs are being introduced to the robotics community almost on a daily basis. Rather than starting ad-hoc HRI studies for each system, or immediately exposing users to live studies within complex scenarios, the use of baseline studies, like the one presented here, will allow for the systematic assessment and identification of important factors for 
the implementation of user friendly HRI.This can be achieved by focusing on the interaction between the users idiosyncratic factors and the behaviour of the robot. In particular, the results show that social expectations may significantly impact interactions with robots, even non-humanoid robots.

Open Access This article is distributed under the terms of the Creative Commons Attribution License which permits any use, distribution, and reproduction in any medium, provided the original author(s) and the source are credited.

\section{References}

1. Asghari Oskoei M, Walters M, Dautenhahn K (2010) An autonomous proxemic system for a mobile companion robot. In: Proceedings of the AISB 2010 symposium on new Frontiers for human robot interaction. AISB

2. Bethel CL, Murphy RR (2007) Non-facial/non-verbal methods of affective expression as applied to robot-assisted victim assessment. In: Proceedings of the ACM/IEEE international conference on human-robot interaction. ACM, pp 287-294

3. Bien ZZ, Lee HE, Do JH, Kim YH, Park KH, Yang SE (2008) Intelligent interaction for human-friendly service robot in smart house environment. Int J Comput Intell Syst 1(1):77-93

4. Burgoon JK, Jones SB (1976) Toward a theory of personal space expectations and their violations. Hum Commun Res 2(2):131-146

5. Cattell RB (1966) The scree test for the number of factors. Multivar Behav Res 1(2):245-276

6. Choi JJ, Kim Y, Kwak SS (2013) The impacts of intergroup relations and body zones on people's acceptance of a robot. In: Humanrobot interaction (HRI), 2013 8th ACM/IEEE international conference on. IEEE, pp 107-108

7. Crowell-Davis SL (2008) Motivation for pet ownership and its relevance to behavior problems. Compendium (Yardley, PA) 30(8):423

8. Dautenhahn K (2007) Socially intelligent robots: dimensions of human-robot interaction. Philos Trans R Soc B Biol Sci 362(1480):679-704

9. Dautenhahn K, Nehaniv CL, Walters ML, Robins B, Kose-Bagci H, Mirza NA, Blow M (2009) KASPAR - a minimally expressive humanoid robot for human-robot interaction research. Appl Bionics Biomech 6(3-4):369-397

10. Dautenhahn K, Walters M, Woods S, Koay KL, Nehaniv CL, Sisbot A, Alami R, Siméon T (2006) How may i serve you? a robot companion approaching a seated person in a helping context. In: Proceedings of the 1st ACM SIGCHI/SIGART conference on humanrobot interaction. ACM, pp 172-179

11. Dautenhahn K, Woods S, Kaouri C, Walters ML, Koay KL, Werry I (2005) What is a robot companion-friend, assistant or butler? In: Intelligent robots and systems. (IROS 2005). 2005 IEEE/RSJ international conference on. IEEE, pp 1192-1197

12. De Santis A, Siciliano B, De Luca A, Bicchi A (2008) An atlas of physical human-robot interaction. Mech Mach Theory 43(3):253270

13. Forlizzi J, DiSalvo C (2006) Service robots in the domestic environment: a study of the roomba vacuum in the home. In: Proceedings of the 1st ACM SIGCHI/SIGART conference on human-robot interaction. ACM, pp 258-265

14. Friedman B, Kahn PH Jr, Hagman J (2003) Hardware companions? What online AIBO discussion forums reveal about the humanrobotic relationship. In: Proceedings of the SIGCHI conference on human factors in computing systems. ACM, pp 273-280
15. Goetz J, Kiesler S, Powers A (2003) Matching robot appearance and behavior to tasks to improve human-robot cooperation. In: Robot and human interactive communication. In: Proceedings. ROMAN 2003. The 12th IEEE international workshop on. IEEE, pp 55-60

16. Green A, Huttenrauch H, Eklundh KS (2004) Applying the wizard-of-Oz framework to cooperative service discovery and configuration. In: Robot and human interactive communication. ROMAN 2004. 13th IEEE international workshop on. IEEE, pp 575-580

17. Hägele M, Schaaf W, Helms E (2002) Robot assistants at manual workplaces: effective co-operation and safety aspects. In: Proceedings of the 33rd ISR (international symposium on robotics) October, vol 7, p 11

18. Hall ET (1969) The hidden dimension. Anchor Books, New York

19. Hayduk LA (1983) Personal space: where we now stand. Psychol Bull 94(2):293

20. Herrmann G, Melhuish C (2010) Towards safety in human robot interaction. Int J Soc Robot 2(3):217-219

21. Hüttenrauch H, Eklundh KS (2002) Fetch-and-carry with CERO: observations from a long-term user study with a service robot. In: Robot and human interactive communication. Proceedings of the 11th IEEE international workshop on. IEEE, pp 158-163

22. Ishiguro H (2006) Android science: conscious and subconscious recognition. Connect Sci 18(4):319-332

23. Jacobsson M (2009) Play, belief and stories about robots: a case study of a pleo blogging community. In: Robot and human interactive communication. ROMAN 2009. The 18th IEEE international symposium on. IEEE, pp 232-237

24. Kaiser HF (1960) The application of electronic computers to factor analysis. Educ Psychol Meas 20(1):141-151

25. Kanda T, Hirano T, Eaton D, Ishiguro H (2004) Interactive robots as social partners and peer tutors for children: a field trial. Hum Comput Interact 19(1):61-84

26. Kendon A (1977) Studies in the behavior of social interaction, vol 6. Humanities Press Intl, Atlantic Highlands, New Jersey

27. Kendon A (1990) Conducting interaction: patterns of behavior in focused encounters, vol 7. CUP Archive

28. Kidd CD, Breazeal C (2007) A robotic weight loss coach. In: Proceedings of the national conference on artificial intelligence, vol 22, p 1985. Menlo Park, CA; Cambridge, MA; London; AAAI Press; MIT Press; 1999

29. Kidd CD, Breazeal C (2008) Robots at home: understanding longterm human-robot interaction. In: Intelligent robots and systems. IROS 2008. IEEE/RSJ international conference on. IEEE, pp 32303235

30. Kim Y, Kwak SS, Kim MS (2012) Am i acceptable to you? effect of a robots verbal language forms on peoples social distance from robots. Comput Hum Behav 29(3):1091-1101

31. Koay K, Lakatos G, Syrdal D, Gácsi M, Bereczky B, Dautenhahn K, Miklósi A, Walters M (2013) Hey! There is someone at your door. A hearing robot using visual communication signals of hearing dogs to communicate intent. In: Artifical life(ALIFE), 2013 IEEE symposium on, Singapore

32. Koay KL, Dautenhahn K, Woods S, Walters ML (2006) Empirical results from using a comfort level device in human-robot interaction studies. In: Proceedings of the 1st ACM SIGCHI/SIGART conference on human-robot interaction. ACM, pp 194-201

33. Koay KL, Sisbot EA, Syrdal DS, Walters ML, Dautenhahn K, Alami R (2007), Exploratory study of a robot approaching a person in the context of handing over an object. In: AAAI spring symposium: multidisciplinary collaboration for socially assistive robotics, pp 18-24

34. Koay KL, Syrdal DS, Walters ML, Dautenhahn K (2007) Living with robots: Investigating the habituation effect in participants' preferences during a longitudinal human-robot interaction study. 
In: Robot and human interactive communication. ROMAN 2007. The 16th IEEE international symposium on. IEEE, pp 564-569

35. Koay KL, Syrdal DS, Walters ML, Dautenhahn K (2009) Five weeks in the robot house-exploratory human-robot interaction trials in a domestic setting. In: Advances in computer-human interactions, 2009. ACHI'09. Second international conferences on. IEEE, pp 219-226

36. Koay KL, Walters ML, May A, Dumitriu A, Christianson B, Burke N, Dautenhahn K (2013) Exploring robot etiquette: refining a HRI home companion scenario based on feedback from two artists who lived with robots in the uh robot house. In: 2013 International conference on social robotics. Springer, Berlin, pp 290-300

37. Kulić D, Croft E (2007) Pre-collision safety strategies for humanrobot interaction. Auton Robots 22(2):149-164

38. Lee SL, Lau IYM, Kiesler S, Chiu CY (2005) Human mental models of humanoid robots. In: Robotics and automation. ICRA 2005. Proceedings of the 2005 IEEE international conference on. IEEE, pp 2767-2772

39. Ljungblad S, Kotrbova J, Jacobsson M, Cramer H, Niechwiadowicz K (2012) Hospital robot at work: something alien or an intelligent colleague? In: Proceedings of the ACM 2012 conference on computer supported cooperative work. ACM, pp 177-186

40. Mutlu B, Forlizzi J (2008) Robots in organizations: the role of workflow, social, and environmental factors in human-robot interaction. In: Human-robot interaction (HRI), 2008 3rd ACM/IEEE international conference on. IEEE, pp 287-294

41. Parlitz C, Hägele M, Klein P, Seifert J, Dautenhahn K (2008) Careo-bot- ${ }^{\circledR}$ - rationale for human-robot interaction design. In: Proceedings of 39th international symposium on robotics (ISR). Seoul, Korea, pp 275-280

42. Pervez A, Ryu J (2008) Safe physical human robot interaction-past, present and future. J Mech Sci Technol 22(3):469-483

43. Powers A, Kramer AD, Lim S, Kuo J, Lee SL, Kiesler S (2005) Eliciting information from people with a gendered humanoid robot. In: Robot and human interactive communication. ROMAN 2005. IEEE international workshop on. IEEE, pp 158-163

44. Reiser U, Jacobs T, Arbeiter G, Parlitz C, Dautenhahn K (2013) Care-o-bot ${ }^{\circledR} 3$-vision of a robot butler. In: Your Virtual Butler. Springer, pp 97-116

45. Robins B, Dautenhahn K, Te Boekhorst R, Billard A (2004) Effects of repeated exposure to a humanoid robot on children with autism. In: Designing a more inclusive world. Springer, Berlin, pp 225-236

46. Saito T, Shibata T, Wada K, Tanie K (2003) Relationship between interaction with the mental commit robot and change of stress reaction of the elderly. In: Computational intelligence in robotics and automation. In: Proceedings of the 2003 IEEE international symposium on, vol 1. IEEE, pp 119-124

47. Sharkey A, Sharkey N (2012) Granny and the robots: ethical issues in robot care for the elderly. Ethics Inf Technol 14(1):27-40

48. Sisbot EA, Alami R, Siméon T, Dautenhahn K, Walters M, Woods S, Koay KL, Nehaniv C (2005) Navigation in the presence of humans. In: Humanoid robots, 2005 5th IEEE-RAS international conference on. IEEE, pp 181-188

49. Smarr CA, Mitzner T, Beer J, Prakash A, Chen T, Kemp C, Rogers W (2013) Domestic robots for older adults: Attitudes, preferences, and potential. Int J Soc Robot, 1-19. doi:10.1007/ s12369-013-0220-0

50. Soyama R, Ishii S, Fukase A (2004) 8 selectable operating interfaces of the meal-assistance device "My Spoon". In: Advances in rehabilitation robotics. Springer, Berlin, pp 155-163

51. Steinert S (2013) The five robots-a taxonomy for roboethics. Int $\mathbf{J}$ Soc Robot, 1-12. doi:10.1007/s12369-013-0221-z

52. Svenstrup M, Tranberg S, Andersen HJ, Bak T (2011) Adaptive human-aware robot navigation in close proximity to humans. Int $\mathrm{J}$ Adv Robot Syst 8(2):1-15
53. Syrdal DS, Dautenhahn K, Walters ML, Koay KL (2008) Sharing spaces with robots in a home scenario-anthropomorphic attributions and their effect on proxemic expectations and evaluations in a live HRI trial. In: Proceedings of the AAAI Fall 2008 symposium "AI in Eldercare: new solutions to old problems", pp 7-9

54. Syrdal DS, Koay KL, Gácsi M, Walters ML, Dautenhahn K (2010) Video prototyping of dog-inspired non-verbal affective communication for an appearance constrained robot. In: ROMAN, 2010 IEEE. IEEE, pp 632-637

55. Syrdal DS, Walters ML, Koay KL, Dautenhahn K (2008) The role of autonomy and interaction type on spatial comfort in an HRI scenario. In: Robotic helpers: user interaction, interfaces and companions in assistive and therapy robotics. Full-day workshop at the third ACM/IEEE human-robot interaction conference (HRI08). University of Hertfordshire

56. Takayama L, Pantofaru C (2009) Influences on proxemic behaviors in human-robot interaction. In: Intelligent robots and systems. IROS 2009. IEEE/RSJ international conference on. IEEE, pp 54955502

57. Tanaka F, Movellan JR, Fortenberry B, Aisaka K (2006) Daily hri evaluation at a classroom environment: reports from dance interaction experiments. In: Proceedings of the 1st ACM SIGCHI/SIGART conference on human-robot interaction. ACM, pp 3-9

58. Torta E, Cuijpers RH, Juola JF (2013) Design of a parametric model of personal space for robotic social navigation. Int J Soc Robot $5(3): 357-365$

59. Wada K, Shibata T (2007) Social effects of robot therapy in a care house-change of social network of the residents for two months. In: Robotics and automation, 2007 IEEE international conference on. IEEE, pp 1250-1255

60. Walters ML, Dautenhahn K, Te Boekhorst R, Koay KL, Syrdal DS, Nehaniv CL (2009) An empirical framework for human-robot proxemics. In: Proceedings of the new Frontiers in human-robot interaction

61. Walters ML, Dautenhahn K, Woods SN, Koay KL (2007) Robotic etiquette: results from user studies involving a fetch and carry task. In: Human-robot interaction (HRI), 2007 2nd ACM/IEEE international Conference on. IEEE, pp 317-324

62. Walters ML, Koay KL, Woods SN, Syrdal DS, Dautenhahn K (2007), Robot to human approaches: Preliminary results on comfortable distances and preferences. In: AAAI spring symposium: multidisciplinary collaboration for socially assistive, robotics, $\mathrm{p}$ 103

63. Walters ML, Lohse M, Hanheide M, Wrede B, Syrdal DS, Koay KL, Green A, Hüttenrauch H, Dautenhahn K, Sagerer G, Severinson Eklund K (2011) Evaluating the robot personality and verbal behavior of domestic robots using video-based studies. Adv Robot 25(18):2233-2254

64. Walters ML, Syrdal DS, Dautenhahn K, Dumitriu A, May A, Christiansen B, Koay KL (2012) My familiar robot companion: preferences and perceptions of CHARLY, a companion humanoid autonomous robot for living with you. In: Advances in autonomous robotics. Springer, Berlin, pp 300-312

65. Walters ML, Syrdal DS, Koay KL, Dautenhahn K, Te Boekhorst R (2008) Human approach distances to a mechanical-looking robot with different robot voice styles. In: Robot and human interactive communication. ROMAN 2008. The 17th IEEE international symposium on. IEEE, pp 707-712

Kheng Lee Koay received his B.Sc. degree in robotics and automated systems and Ph.D. degree from the University of Plymouth, U.K. in 1997 and 2003, respectively. He is currently a Senior Research Fellow at the Adaptive Systems Research Group at the University of 
Hertfordshire, U.K. His research interests include Mobile Robotics, Robotic Home Companion and Human-Robot Interaction especially in the aspect of human-centred socially acceptable interaction, design and evaluation methodology. He was involved in several European projects Cogniron (Cognitive Robot Companion), LIREC (Living with Robots and Interactive Companions) and is currently working in the FP7 European project ACCOMPANY (Acceptable Robotics Companions for Ageing Years) and EPSRC project Trustworthy Robotic Assistants.

Dag Sverre Syrdal received his B.Sc. in Psychology from Queen's University Belfast in 2001 and his M.Sc. in Research Methods and Data Analysis from the University of Hertfordshire. He joined the Adaptive Systems Research Group at the University of Hertfordshire in 2006, where his work has centred around the planning, execution and analysis of user studies within the EU FP6/7 Cogniron, LIREC and ACCOMPANY projects. His research interests include Human-Robot Interaction with an emphasis on socially acceptable interactions in humancentred environments, as well as the role of Individual Differences in HRI.

Mohammadreza Ashgari-Oskoei is Assistant Professor at the University of Allameh Taba-tabai (Iran) and a Visiting Researcher in University of Essex (UK). He received his MS in Control System Engineering from the University of Tehran (Iran) in 1993 and his $\mathrm{PhD}$ in Computer Science from the University of Essex in 2009. He was a member of the EU research project (LIREC) in University of Hertfordshire (2009-2012). His research interests include developing a computational framework for human robot proxemics, robot sensor fusion, vision and navigation.
Michael L. Walters received his H.N.D. in Computer Aided Engineering in 1988, M.Sc. in Artificial Neural Networks and Robotics in 1993 and his PhD in 2008. He is currently a Lecturer in the School of Computer Science at the University of Hertfordshire. His research interests include Human-Robot Interaction and social robotics, in particular how robots should behave when interacting with humans and working in a human environment.

Kerstin Dautenhahn is Full Professor in the School of Computer Science at University of Hertfordshire in U.K. where she coordinates the Adaptive Systems Research Group. She has pioneered research in social robotics, human-robot interaction, assistive technology and published more than 300 research articles. Prof. Dautenhahn has edited several books and frequently gives invited keynote lectures at international meetings. She organizes international conferences and has been Principal Investigator of her research team in several European FP5/6/7 projects. Prof. Dautenhahn is Founding Editor in Chief of the Journal of Interaction Studies: Social Behaviour and Communication in Biological and Artificial Systems, as well as Associate Editor of several other international journals. 